\title{
2-(3-Pyrrolin-1-yl)-1,4-naphthoquinones: Photoactivated Alkylating Agents
}

\author{
Aaron Aponick, *[a][*] Amber L. Dietz, ${ }^{[\mathrm{a}]}$ and William H. Pearson ${ }^{[\mathrm{a}][+] 1}$
}

Keywords: Quinones / Pyrroline / Michael addition / Alkylation / Photoactivation

The preparation of 2-(3-pyrrolin-1-yl)-1,4-naphthoquinones and a study of their use as photoactivated alkylating agents is reported. The title compounds were easily synthesized by conjugate addition of the corresponding 3-pyrrolines to various naphthoquinones. Upon exposure to ambient room light, the compounds undergo an internal redox reaction to form 2-(pyrrol-1-yl)-1,4-hydroquinones, which are activated for nucleophilic addition by an $\mathrm{S}_{\mathrm{N}} 1$ azafulvene mechanism. Control experiments demonstrated that the redox reaction is triggered by light and that the nucleophilic addition does not proceed before this activation occurs.

(@) Wiley-VCH Verlag GmbH \& Co. KGaA, 69451 Weinheim, Germany, 2008)

\section{Introduction}

Many cancer chemotherapy strategies involve drugs that approach the toxicity level to be effective. Because cancerous cells proliferate more rapidly than normal cells, one general chemotherapeutic strategy is to stop the cell-division process by targeting DNA. During cell division, a DNA polymerase reads one strand, which it has separated from the double helix, then synthesizes its complement by relying on Watson-Crick base pairing of an incoming nucleotide triphosphate. Thus, if the two strands cannot be separated, replication is not possible. Reagents that react with DNA have been shown to form both inter- and intrastrand crosslinks as well as interhelix cross-links and DNA-protein cross-links. ${ }^{[1]}$ In general, interstrand cross-links are thought to be the most effective in disrupting this process.

An example of a natural product that cross-links DNA is illustrated in Scheme 1. Moncrotaline (1) is a carcinogenic pyrrolizidine alkaloid isolated from plant material in the pyrroline oxidation state. ${ }^{[2]}$ Upon oxidation by P450 enzymes, the highly reactive pyrrole $\mathbf{2}$ is formed. ${ }^{[3]}$ This species is the active cross-linker and after two elimination/addition sequences forms the ultimate adduct 3 . In addition to pyrrolizidine alkaloids like monocrotoline, other naturally occurring molecules such as mitomycin $\mathrm{C}$ metabolites and

[a] Department of Chemistry, University of Michigan, Ann Arbor, MI 48109-1055, USA

[\$] Current address: Department of Chemistry, University of Florida,

Gainesville, FL 32611, USA

Fax: +1-352-846-0296

E-mail: aponick@chem.ufl.edu

[†t]Current address: Berry \& Associates, Inc.

2434 Bishop Circle East, Dexter, MI 48130, USA

Supporting information for this article is available on the WWW under http://www.eurjoc.org or from the author. synthetic molecules that share the reactive pyrrole core of $\mathbf{2}$, generically represented by $\mathbf{4}$, have been shown to crosslink DNA. ${ }^{[4]}$ Unfortunately, these compounds (both synthetic and natural) often prove to be too reactive as they have the potential to alkylate a multitude of different chemical species.

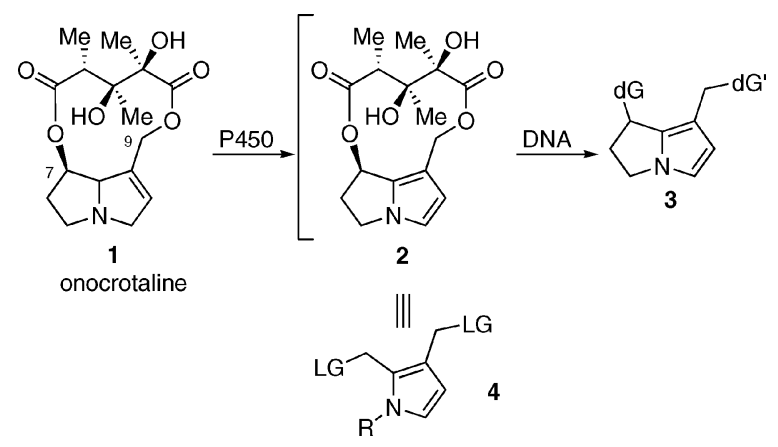

Scheme 1. DNA cross-linking by monocrotaline. $\mathrm{LG}=$ leaving group.

Toxicity also plagues these compounds because there is no specificity for tumor cells over healthy cells. Anderson, Hopkins, and several other groups, have tried to circumvent the problems of reactivity and stability in water by synthesizing various analogs, but this has met with only limited success. ${ }^{[5]}$

To solve these issues, nature has built in a control element with the P450 enzyme activation of monocrotaline (1) to activate the C-7 and C-9 positions. Chemists have also added their own control elements by modifying natural products to contain groups such as photolabile protecting 
groups that activate the molecule by deprotection of a reactive functional group. Two elegant examples of this have been reported by Williams in which reactive analogues of the natural products monocrotaline ${ }^{[6]}$ and FR900482 $2^{[7]}$ are released after deprotection of a 6-nitroveratryl carbamate (NVOC). This concept retains the natural product-like structure that is responsible for the selective reaction with DNA, but is limited due to the necessity of a natural product starting material. An ideal molecule would be both selective and easily prepared so that the binding could be modified to select target different sequences.

We sought to combine the idea of a reactive pyrrole such as $\mathbf{4}$ with the notion of a triggering event (e.g., light) that would lead to its generation from a stable starting material. A search of the literature for the photochemical generation of pyrroles revealed several interesting examples. ${ }^{[8]}$ Maruyama reported that irradiation of a benzene solution of the quinone 5 with a mercury arc lamp produced the hydroquinone 6 (Scheme 2). ${ }^{[8]}$ An internal redox reaction provided the hydroquinone with concomitant oxidation of the pyrroline moiety to the pyrrole as evidenced by trapping $\mathbf{6}$ as the diacetate in degassed solutions. In experiments where the solvent was not degassed, the hydroquinone 6 was oxidized to a pyrrole-bearing quinone. Franck reported the production of a pyrrole-bearing quinone from a benzoquinone and a 3-pyrroline, though the potential role of ambient light was not noted. ${ }^{[8 c]}$

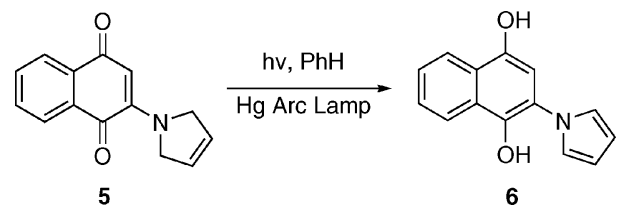

Scheme 2. Internal redox reactions of pyrroline-substituted quinones.

With evidence for a photochemically generated pyrrole, we surmised that the quinones 7 , with structures similar to 5 except now bearing one or more leaving groups (LG), could provide a platform to explore the preparation of simplified alkylating agents. Recent advances in the use of fiber optics and lasers in medicine ${ }^{[9]}$ make photochemically activated alkylating agents attractive targets because they can be introduced in an inactive form and "turned on" at will. The quinones $\mathbf{7}$ should not be good alkylating agents until the photochemical redox reaction takes place to afford the pyrroles 4 . At this point, the deactivating quinone nucleus has been reduced to an electron-rich hydroquinone, and the pyrroline ring has been oxidized to a pyrrole, making the substitution reactions facile by the intervention of azafulvene intermediates. In addition, the quinones 7 should be easily prepared by conjugate addition of the requisite 3pyrroline to a quinone (Figure 1). This disconnection should lead to a facile assembly of a diverse array of compounds with varied 3-pyrroline and quinone fragments. Our initial studies to explore the preparation and reactivity of this potential scaffold are reported herein.

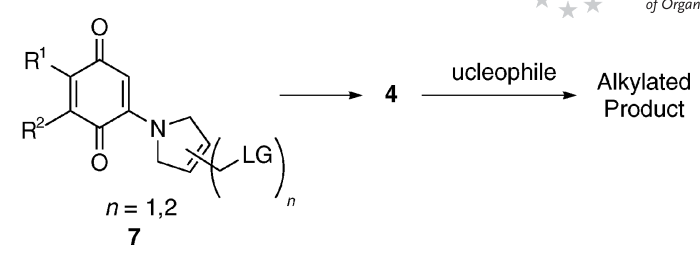

Figure 1. General design of potential photochemically triggered alkylating agents.

\section{Results and Discussion}

To prepare the target compounds, a variety of 3-pyrrolines (e.g., 8-15) was necessary in order to find the best choices for the leaving group and its position on the ring for successful photochemically triggered alkylation chemistry. We examined both mono- and bis(alkylators) (Figure 2).

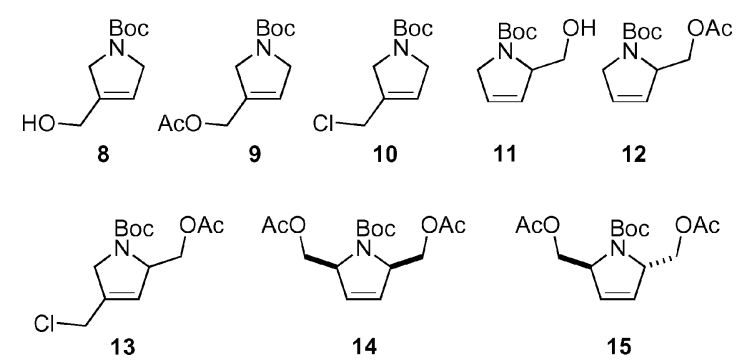

Figure 2. Desired substituted 3-pyrrolines.

The 3-substituted pyrrolines were prepared by a ringclosing metathesis (RCM) strategy (Scheme 3). ${ }^{[10]}$ The necessary dienes were prepared in a straightforward manner starting with alkylation of $N$-Boc-allylamine (16) with commercially available 3-chloro-2-(chloromethyl)-1-propene (17). The chloride was then displaced with acetate in $89 \%$ yield, and both 18 and 19 were cyclized in good yield by using Grubbs' first-generation catalyst ${ }^{[10]}$ without incident.

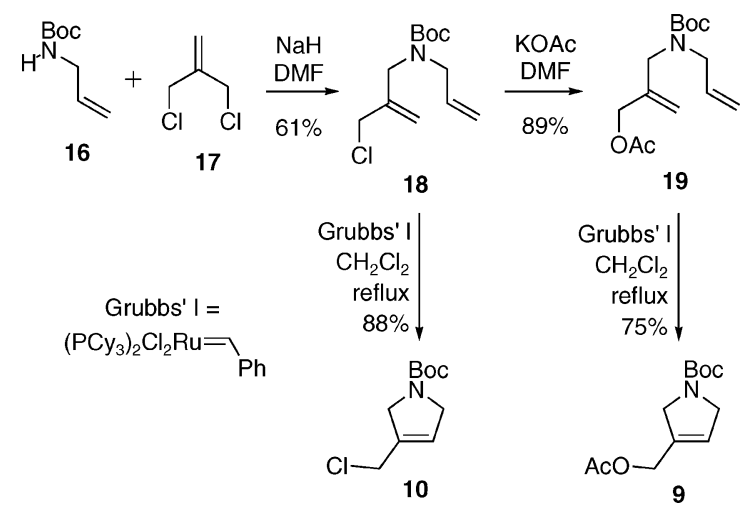

Scheme 3. Synthesis of 3-substituted-3-pyrrolines by RCM.

To prepare the 2-substituted 3-pyrroline 12, the procedure of Blechert was applied to give $\mathbf{1 1}^{[11]}$ starting with the known $N$-protected vinylglycine 20 ${ }^{[12]}$ (Scheme 4). Re- 
duction of the methyl ester $\mathbf{2 0}$ and formation of the oxazolidinone $\mathbf{2 2}$ proceeded in good yield. Hydrolysis and Boc protection then gave the necessary diene $\mathbf{2 3}$ in $82 \%$ yield. RCM by using Grubbs' first-generation ruthenium catalyst provided $\mathbf{1 1}$ and $\mathbf{1 2}$ after acylation.

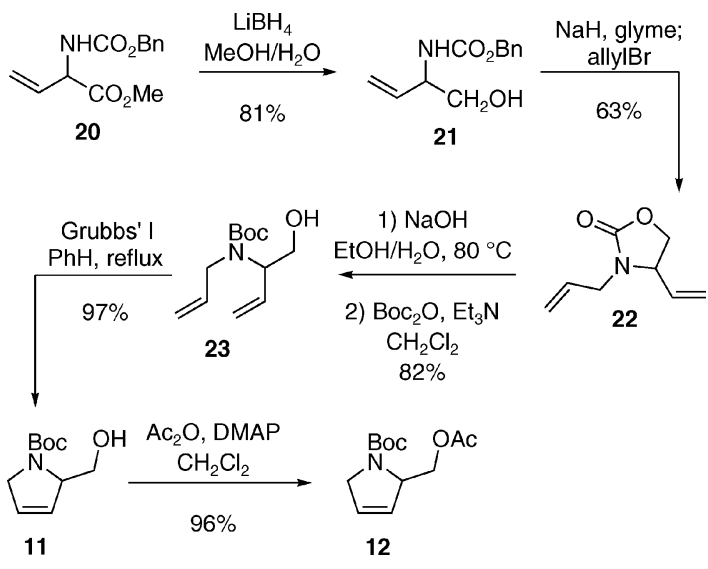

Scheme 4. Synthesis of 2-substituted-3-pyrrolines $\mathbf{1 1}$ and $\mathbf{1 2}$.

The necessary disubstituted analogs were prepared in the following manner. $N$-Boc-vinylglycinol, derived from 21, was protected as its triisopropylsilyl ether (Scheme 5). The second allyl unit was then introduced by alkylation with the allyl chloride 17, and the TIPS group was removed with TBAF in THF, all in good yield. RCM with Grubbs' second generation catalyst ${ }^{[10]}$ provided 28 in $98 \%$ yield and acylation provided the desired 3-pyrroline $\mathbf{1 3}$.

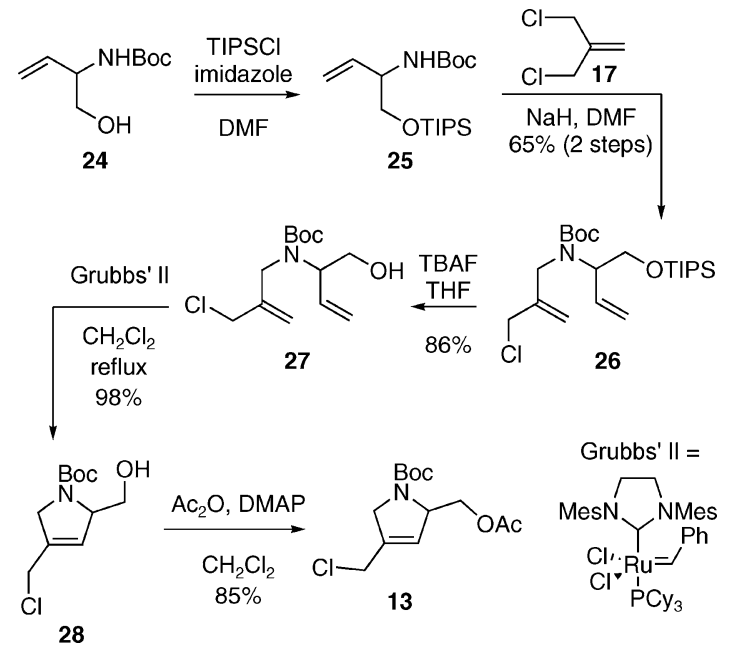

Scheme 5. Synthesis of 2,4-disubstituted-3-pyrrolines 28 and 13.

The 3-pyrrolines 14 and $\mathbf{1 5}$ were obtained from 29 and 30 in a straightforward manner by reduction and acylation (Scheme 6). The known diesters 29 and $\mathbf{3 0}$ were prepared from the corresponding pyrroles by taking advantage of Donohoe's flexible partial reduction method. ${ }^{[13]}$
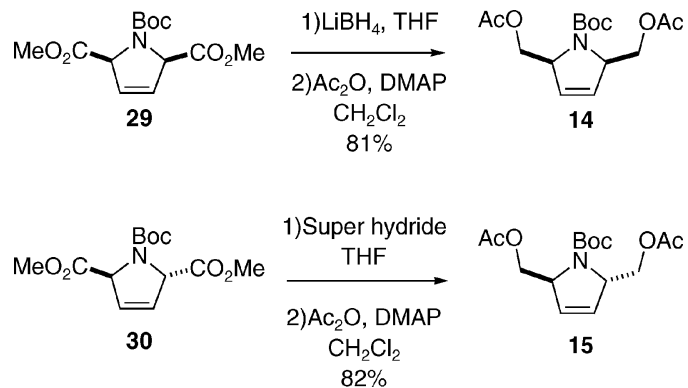

Scheme 6. Synthesis of 2-substituted-3-pyrrolines $\mathbf{1 4}$ and $\mathbf{1 5}$

With the 3-pyrrolines in hand we next set out to prepare a variety of the target quinones represented by the generic structure 7. Deprotection of the $N$-Boc groups of the pyrrolines and in situ neutralization of the TFA salts followed by addition of the desired quinone and $\mathrm{CuBr}$ proceeded in reasonable yield (Table 1). ${ }^{[14]}$ This reaction was used to prepare the naphthoquinones $\mathbf{3 4} \mathbf{4 1}$, juglones $\mathbf{4 2 - 4 3}$, isoquinolinequinones $\mathbf{4 4} \mathbf{4 5}$, and the quinolinequinones $46-$ 50. It is important to note that these reactions were carried out in the absence of light to avoid unwanted redox reactions as the preparation of quinone 5 (Scheme 2) in our laboratories showed that the photochemical reaction proceeds in ambient room light and that the mercury arc lamp reported is unnecessary.

With a good indication that the photochemical redox reaction would proceed (vide supra), the next goal was to optimize the redox reaction and determine if the desired alkylation was possible. To this end, initial NMR-tube experiments were conducted in $\mathrm{CDCl}_{3}$ as outlined in Scheme 7. It is desirable to avoid the use of a mercury arc lamp to trigger the redox reaction because it would be more convenient to use a sunlamp, but this would also provide less intense irradiation of the reaction. These irradiation conditions were tested, and when quinone $\mathbf{3 5}$ was irradiated with a sunlamp in the presence of ethanethiol, only the pyrrole 51 was observed by ${ }^{1} \mathrm{H}$ NMR spectroscopy. The internal redox reaction to $\mathbf{3 5 a}$ had been successful, but presumably further oxidation by oxygen in the system had occurred instead of the desired alkylation.

It is known that the presence of electronegative substituents on the pyrrole nitrogen atom suppresses the azafulvene mechanism of substitution, which explains why the pyrroloquinones $\mathbf{5 1}$ and $\mathbf{5 2}$ do not react with ethanethiol. ${ }^{[15]}$ Because the reaction had proceeded rapidly $(15 \mathrm{~min})$ and yielded no evidence of the hydroquinone, it was postulated that premature air oxidation of the desired hydroquinone to the quinone oxidation state was preventing alkylation. Experiments with ambient light were carried out with the hope that a less intense light source at lower temperature might lengthen the lifetime of the hydroquinone. Unfortunately, while the quinone $\mathbf{3 4}$ underwent the internal redox reaction, the resultant hydroquinone was again oxidized to the quinone $\mathbf{5 2}$ before alkylation could occur. Additionally, irradiation of degassed solutions of $\mathbf{3 4}$ yielded only intractable mixtures. 
Table 1. Michael addition of 3-pyrrolines.

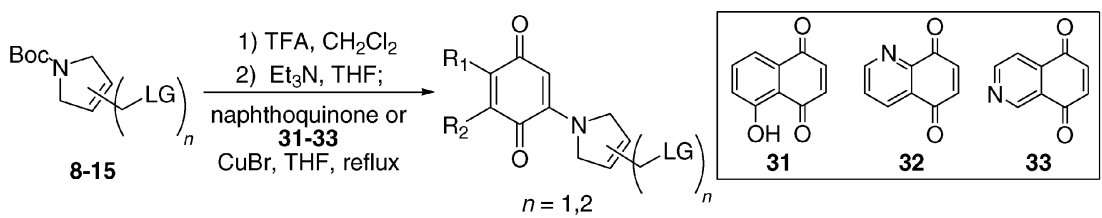

34-50

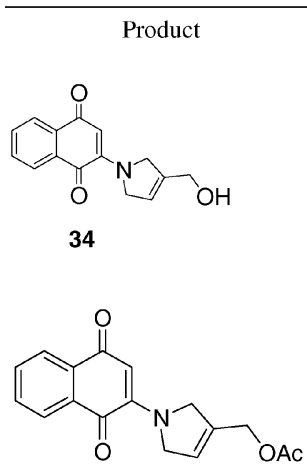

35<smiles>O=C1C=C(N2CC=C(CCl)C2)C(=O)c2ccccc21</smiles>

36<smiles>O=C1C=C(N2CC=CC2CO)C(=O)c2ccccc21</smiles>

37<smiles>CC(=O)OCC1C=CCN1C1=CC(=O)c2ccccc2C1=O</smiles><smiles>CC(=O)OCC1C=C(CCl)CN1C1=CC(=O)c2ccccc2C1=O</smiles>

$61^{[\mathrm{a}]}$<smiles>CC(=O)OCC1C=CC(COC(C)=O)N1C1=CC(=O)c2ccccc2C1=O</smiles>

52<smiles>CC(=O)OC[C@@H]1C=C[C@@H](C)N1C1=CC(=O)c2ccccc2C1=O</smiles>

OAc

51<smiles>CC(=O)OCC1C=CCN1C1=CC(=O)c2cccc(O)c2C1=O</smiles>

42<smiles>O=C1C=C(N2CC=C(CCl)C2)C(=O)c2c(O)cccc21</smiles>

43

50<smiles>CC(=O)OCC1C=CCN1C1=CC(=O)c2ccncc2C1=O</smiles>

44<smiles>CC(C)(F)[C@H]1CCN(C2=CC(=O)c3ccncc3C2=O)C1</smiles>

52<smiles>CC(=O)OCC1C=CCN1C1=CC(=O)c2ncccc2C1=O</smiles>

46<smiles>O=C1C(N2CC=C(CCl)C2)=CC(=O)c2ncccc21</smiles>

47<smiles>CC(=O)OCC1C=C(CCl)CN1C1=CC(=O)c2ncccc2C1=O</smiles>

48<smiles>CC(=O)OC[C@@H]1C=C[C@H](COC(C)=O)N1C1=CC(=O)c2ncccc2C1=O</smiles>

51<smiles>CC(=O)OC[C@@H]1C=C[C@H](COC(C)=O)N1C1=CC(=O)c2ncccc2C1=O</smiles>
$0^{[\mathrm{h}, \mathrm{e}]}$ 55

[a] Conditions: (1) TFA, $\mathrm{CH}_{2} \mathrm{Cl}_{2}$. (2) $\mathrm{NH}_{4} \mathrm{OH}, \mathrm{MeOH}$; naphthoquinone, CuBr, $\mathrm{Et}_{2} \mathrm{O}$. [b] Conditions: (1) $\mathrm{TFA} \mathrm{CH}_{2} \mathrm{Cl}_{2}$. (2) Et $\mathrm{N}_{3}$, THF; quinolinequinone, $\mathrm{CeCl}_{3} \cdot 7 \mathrm{H}_{2} \mathrm{O}, \mathrm{EtOH}$. [c] The compound was found to be unstable to storage for prolonged periods. [d] Variable results were observed giving $0-12 \%$ yield, but in any case the reaction was not preparatively useful. [e] Decomposition.

Fortunately, a combination of a better leaving group and less intense irradiation provided an encouraging result. When the acetate $\mathbf{3 5}$ was treated with ethanethiol in the presence of ambient room light, a small amount of the desired alkylated product was isolated along with $53 \%$ of the non-alkylated product $\mathbf{5 1}$ (Scheme 8). Moving to a better leaving group, the (chloromethyl)-3-pyrroline $\mathbf{3 6}$ gave the desired sulfide 53 in $54 \%$ yield. In both cases, we did not observe the presumed hydroquinone species.

In order to determine whether the proposed hydroquinone-pyrroline intermediate was responsible for the successful alkylation, we carried out several experiments. Con- 
<smiles>CC(=O)OCC1=CCN(C2=CC(=O)c3ccccc3C2=O)C1</smiles><smiles>CCCCCCOCc1ccn(-c2cc(O)c3ccccc3c2O)c1</smiles><smiles>CC(=O)OCc1ccn(C2=C(C)C(=O)c3ccccc3C2=O)c1</smiles><smiles>O=C1C=C(N2CC=C(CO)C2)C(=O)c2ccccc21</smiles><smiles>CCCCCC(=O)OCC</smiles><smiles>O=C1C=C(n2ccc(CO)c2)C(=O)c2ccccc21</smiles>

Scheme 7. Initial irradiation studies.<smiles>CC(=O)OCC1=CCN(C2=CC(=O)c3ccccc3C2=O)C1</smiles><smiles>CCSCc1ccn(C2=CC(=O)c3ccccc3C2=O)c1</smiles><smiles>O=C1C=C(N2CC=C(CCl)C2)C(=O)c2ccccc21</smiles><smiles>ClC1CCCCC1</smiles><smiles>CCCCCCCCCc1ccn(C2=CC(=O)c3ccccc3C2=O)c1</smiles>

Scheme 8. Initial alkylation studies.

trol experiments excluding light from the reaction under conditions otherwise identical led to only the observation of the starting materials $\mathbf{3 5}$ and $\mathbf{3 6}$, demonstrating that no chemistry can occur prior to light activation. To probe the issue of whether the alkylation occurs before or after oxidation of the hydroquinone to the quinone, the 3-pyrroline $\mathbf{3 6}$ was oxidized to the pyrrole $\mathbf{5 4}$ and exposed to the alkylation reaction conditions (Scheme 9). The pyrrole 54 failed to undergo a significant amount of alkylation $(<10 \%)$ indicating that while $\mathbf{5 4}$ may participate in the reaction to some extent that it is likely a minor pathway and that the corresponding hydroquinone is the most likely reactive species.

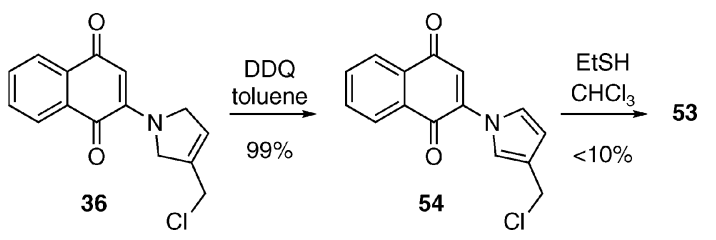

Scheme 9. Control experiment to determine when alkylation occurs.
Table 2. Optimization studies.<smiles>CC(C)Cc1ccn(C2=CC(=O)c3ccccc3C2=O)c1</smiles>

\begin{tabular}{|c|c|c|c|c|c|}
\hline Entry $^{[\mathrm{a}]}$ & Solvent & Base & $h v$ & Time & Yield \\
\hline 1 & toluene & - & sunlamp & $1 \mathrm{~h}$ & $22 \%$ \\
\hline 2 & $\mathrm{CH}_{2} \mathrm{Cl}_{2}$ & - & sunlamp & $1 \mathrm{~h}$ & $26 \%$ \\
\hline 3 & toluene & - & ambient & $3 \mathrm{~d}$ & $40 \%$ \\
\hline 4 & $\mathrm{CH}_{3} \mathrm{CN}$ & - & ambient & $1 \mathrm{~d}$ & $34 \%$ \\
\hline 5 & $\mathrm{CHCl}_{3}$ & - & ambient & $1 \mathrm{~d}$ & $54 \%$ \\
\hline 6 & $\mathrm{CHCl}_{3}$ & $\mathrm{~K}_{2} \mathrm{CO}_{3}$ & ambient & $2 \mathrm{~d}$ & $51 \%$ \\
\hline 7 & $\mathrm{CHCl}_{3}$ & $\begin{array}{l}\text { 2,6-luti- } \\
\text { dine }\end{array}$ & ambient & $2 d$ & $63 \%$ \\
\hline 8 & $\mathrm{MeOH}$ & - & ambient & $3 d$ & $0 \%$ \\
\hline 9 & $\mathrm{MeOH}$ & $\begin{array}{l}\text { 2,6-luti- } \\
\text { dine }\end{array}$ & ambient & $1 \mathrm{~d}$ & $0 \%$ \\
\hline
\end{tabular}

[a] 10 mol-equiv. of EtSH employed.<smiles>CC(=O)OCC1C=CCN1C1=CC(=O)c2ccccc2C1=O</smiles><smiles>[CH][C@@H](C)[C@@H](O)C(Cl)Cl</smiles><smiles>CC[SeH2]Cc1cccn1C1=CC(=O)c2ccccc2C1=O</smiles><smiles>O=C1C(N2CC=C(CCl)C2)=CC(=O)c2ncccc21</smiles>

$\mathrm{EtSH}, \mathrm{CHCl}_{3}$ 2,6-Iutidine

ambient light $42 \%$<smiles>CC(O)(O)CC1C=CCN1C1=CC(=O)c2ncccc2C1=O</smiles>

$\mathrm{EtSH}, \mathrm{CHCl}_{3}$

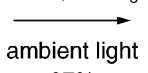

$67 \%$<smiles>CCCCCCc1ccn(-c2cc(O)c3ncccc3c2O)c1</smiles><smiles>[B]Cc1cccn1-c1cc(O)c2ncccc2c1O</smiles><smiles>CC(=O)OCC1C=CCN1C1=CC(=O)c2cccc(O)c2C1=O</smiles>

$\mathrm{EtSH}, \mathrm{CHCl}_{3}$

$\overrightarrow{\text { ambient light }}$

$44 \%$<smiles>CC[Se]Cc1cccn1C1=CC(=O)c2cccc(O)c2C1=O</smiles><smiles>COCC1C=CCN1C1=CC(=O)c2ccncc2C1=O</smiles>

$\mathrm{EtSH}, \mathrm{CHCl}$

$\overrightarrow{\text { ambient light }}$ no reaction<smiles>Oc1cc(N2CC=C(CCl)C2)c([SH3+])c2cnccc12</smiles>

$\mathrm{EtSH}, \mathrm{CHCl}_{3}$ $\stackrel{\text { 2,6-lutidine }}{\longrightarrow}$ no reaction

Scheme 10. Light-triggered alkylation reactions of mono(electrophiles). 
The reaction was next optimized for light source, solvent, time, and additive (Table 2). It was found that the best yield was obtained by using ambient light in chloroform with 2,6lutidine as an additive to remove $\mathrm{HCl}$ generated during the reaction. To provide the best mimic for biological systems, it was hoped that the reaction would function in a polar protic solvent, but unfortunately the use of methanol provided no product.

With these conditions established, alkylation studies with the remaining monoalkylators are outlined in Scheme 10. Alkylation of the naphthoquinone $\mathbf{3 8}$ with the electrophilic group in the 2-position proceeded in $86 \%$ yield without any added base. Likewise, alkylation of the quinolinequinones 47 and 48 showed the same trend. The 2-substituted 3pyrroline reacted substantially better, although it is interesting that the hydroquinone oxidation states of these compounds are stable enough to be isolated without further oxidation to the corresponding quinones, further validating our hypothesis that hydroquinones are involved in the alkylation. The juglone $\mathbf{4 2}$ also functioned in the reaction, but the isoquinolinequinones $\mathbf{4 4}$ and $\mathbf{4 5}$ failed to undergo even the initial internal redox reaction.

Alkylation of the bis(electrophiles) 39, 40, 48, and 49 were studied with hopes that the sequence involving redox, two ionization/alkylation steps, and oxidation to the quinone would be as efficient as the alkylation reactions described in Scheme 10. As outlined in Scheme 11, the chemistry worked as planned, but the products of the reactions employing naphthoquinones $\mathbf{3 9}$ and $\mathbf{4 0}$ were unusual in that<smiles>CC(=O)OCC1C=C(CCl)CN1C1=CC(=O)c2ccccc2C1=O</smiles>

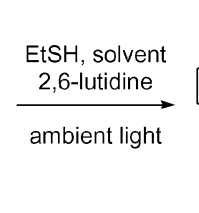<smiles>CC(=O)OCC1C=CC2COC(=O)C(N3C(=O)C(=O)c4ccccc4C3=O)=CC12</smiles>

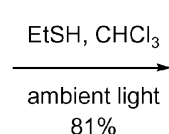<smiles>CC(=O)OCC1C=C(CCl)CN1C1=CC(=O)c2ncccc2C1=O</smiles>

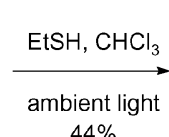<smiles>CC(=O)OCC1C=CC2COC(=O)C(N3C(=O)C(=O)c4ncccc4C3=O)C12</smiles>
$81 \%$ $44 \%$

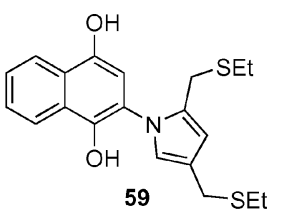

solvent $=\mathrm{CHCl}_{3} \quad 40 \%$ solvent $=\mathrm{CH}_{3} \mathrm{CN} 76 \%$

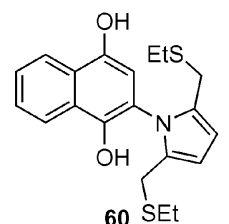

$60 \mathrm{SEt}$

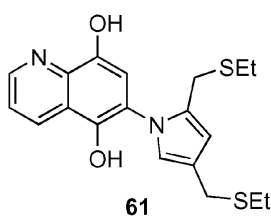

$\mathrm{EtSH}, \mathrm{CHCl}_{3}$

ambient light $30 \%$

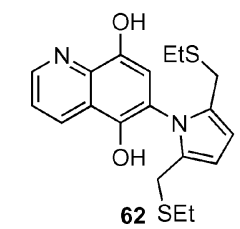

Scheme 11. Light-triggered alkylation reactions of bis(electrophiles). the hydroquinones $\mathbf{5 9}$ and $\mathbf{6 0}$ were isolated and characterized. These compounds were found to slowly undergo oxidation to the corresponding quinones, but were characterized in the hydroquinone oxidation state. It was gratifying that these two reactions proceeded in nearly $80 \%$ for each, demonstrating that the designed sequence could be very efficient.

\section{Conclusions}

A diverse array of 2-(3-pyrrolin-1-yl)-1,4-naphthoquinones were prepared by Michael addition of the corresponding 3-pyrrolines with the requisite quinones. The reaction was found to be quite general and effective when carried out in the absence of light. The target compounds were observed to undergo an internal redox reaction triggered by ambient room light to generate reactive pyrroles that are then alkylated by ethanethiol. This provides a proof of principle for the potential use of these compounds as photoactivated alkylating agents.

\section{Experimental Section}

General: Unless otherwise noted, reactions were carried out under dry nitrogen in flame-dried glassware equipped with tightly fitted rubber septum. Toluene, triethylamine, pyridine, dichloromethane, diethylamine, and diisopropylamine were distilled from powdered calcium hydride. Dimethylformamide (DMF) was distilled at reduced pressure from barium oxide. Tetrahydrofuran (THF) and diethyl ether were distilled under nitrogen from sodium/benzophenone ketyl. Commercial $n$-butyllithium was titrated with diphenylacetic acid prior to use. Reagents which required preparation according to literature procedures are referenced when described. All other reagents were obtained from Aldrich Chemical Company, and used without further purification. Chromatography refers to liquid chromatography on silica gel. Proton nuclear magnetic resonance $\left({ }^{1} \mathrm{H}\right.$ NMR) spectra were recorded at 500,400 or $300 \mathrm{MHz}$ respectively, with Varian Unity INOVA 500, Varian Unity INOVA 400 or Varian Mercury 300 spectrometers. Chemical shifts $(\delta)$ are reported in parts per million (ppm) downfield relative to tetramethylsilane (TMS). Coupling constants $(J)$ are reported in Hz. Multiplicities are reported by using the following abbreviations: s, singlet; $\mathrm{d}$, doublet; t, triplet; q, quartet; $\mathrm{m}$, multiplet; br., broad. ${ }^{13} \mathrm{C} \mathrm{NMR}$ spectra were recorded with a Varian Unity INOVA 400 spectrometer at $100 \mathrm{MHz}$, and chemical shifts are reported in ppm relative to the carbon resonance of $\mathrm{CDCl}_{3}(\delta=77.06 \mathrm{ppm})$. Infrared (IR) spectra were obtained with a Perkin Elmer Spectrum BX FT-IR spectrometer at $2.0 \mathrm{~cm}^{-1}$ resolution and are reported in wavenumbers (signal intensity and shape). The following abbreviations are used in describing IR data: w, weak; m, medium; s, strong; br., broad. UV/Vis spectra were recorded with a Shimadzu UV160U spectrophotometer. Routine mass spectrometric data were obtained by using a Hewlett Packard 6890 gas chromatograph equipped with an HP 5973 mass-selective detector (GCMS) by electron impact (EI) at $70 \mathrm{eV}$. High resolution mass spectral (HRMS) and elemental analyses were performed by the facilities operated by the University of Michigan.

tert-Butyl Allyl[2-(chloromethyl)allyl]carbamate (18): $N$-Boc-Allylamine (16) $(0.157 \mathrm{~g}, 1.00 \mathrm{mmol})$ and 3-chloro-2-(chloromethyl)-1propene $(0.125 \mathrm{~g}, 1.00 \mathrm{mmol})$ were dissolved in DMF $(2.5 \mathrm{~mL})$. 
$\mathrm{NaH}(0.06 \mathrm{~g}$ of a $60 \%$ dispersion in light mineral oil, $1.5 \mathrm{mmol})$ was added to the clear colorless solution which immediately turned to a cloudy pale yellow. After $1 \mathrm{~h}$, the reaction was quenched by dropwise addition of $\mathrm{H}_{2} \mathrm{O}(25 \mathrm{~mL})$, then diluted with diethyl ether (ca. $25 \mathrm{~mL}$ ). The layers were separated, and the organic layer was washed successively with three portions of $\mathrm{H}_{2} \mathrm{O}$, then brine, dried $\left(\mathrm{MgSO}_{4}\right)$, and the solvent removed in vacuo. Chromatography (0$6 \%$ EtOAc/hexane gradient) afforded $0.15 \mathrm{~g}(61 \%)$ of a colorless oil. $R_{f}=0.29$ (10\% EtOAc/hexanes). ${ }^{1} \mathrm{H}$ NMR (400 MHz, $\left.\mathrm{CDCl}_{3}\right)$ : $\delta=5.82-5.72(\mathrm{~m}, 1 \mathrm{H}), 5.27(\mathrm{~s}, 1 \mathrm{H}), 5.16-5.05(\mathrm{~m}, 3 \mathrm{H}), 4.03(\mathrm{~s}$, $2 \mathrm{H}), 3.93$ (s, $2 \mathrm{H}), 3.82-3.78(\mathrm{~m}, 2 \mathrm{H}) 1.46$ (s, $9 \mathrm{H}) \mathrm{ppm} .{ }^{13} \mathrm{C} \mathrm{NMR}$ $\left(100 \mathrm{MHz} \mathrm{CDCl}_{3}\right): \delta=155.5,141.3,133.6,116.9,116.0,80.0,48.9$, 48.3, 45.7, 28.4. IR (neat): $\tilde{v}=3083$ (w), 2978 (m), 2930 (m), 1697 (s), 1645 (m), 1457 (s), 1404 (s), 1366 (s) cm $\mathrm{cm}^{-1}$ LRMS (EI, $70 \mathrm{eV}$ ): $\mathrm{m} / \mathrm{z}(\%)=245$ (0.036), 210 (2), 189 (17), 172 (19), 154 (100), 110 (32), 57 (78). HRMS (CI with $\mathrm{NH}_{3}$ ): calcd. for $\mathrm{C}_{12} \mathrm{H}_{21} \mathrm{ClNO}_{2}[\mathrm{M}$ $+\mathrm{H}]^{+}$246.1261; found 246.1249. $\mathrm{C}_{12} \mathrm{H}_{20} \mathrm{ClNO}_{2}$ (245.12): calcd. C 58.65, H 8.20, N 5.70; found C 58.63, H 8.20, N 5.47.

tert-Butyl [2-(Acetoxymethyl)allyl]allylcarbamate (19): Compound $18(0.154 \mathrm{~g}, 0.630 \mathrm{mmol})$ was dissolved in DMF $(1.6 \mathrm{~mL})$, and to the solution was added KOAc $(0.068 \mathrm{~g}, 0.69 \mathrm{mmol})$. The reaction mixture was heated to $65^{\circ} \mathrm{C}$ for $3.5 \mathrm{~h}$, cooled to room temp., then diluted with water $(10 \mathrm{~mL})$ and diethyl ether $(10 \mathrm{~mL})$. The layers were separated, and the organic layer was washed successively with three portions of $\mathrm{H}_{2} \mathrm{O}$, then brine, dried $\left(\mathrm{MgSO}_{4}\right)$, and the solvent removed in vacuo to yield $0.155 \mathrm{~g}(91 \%)$ of a clear colorless oil which was typically used without further purification. An analytically pure sample was obtained by chromatography (5\% EtOAc/ hexanes). $R_{f}=0.16\left(10 \%\right.$ EtOAc/hexanes). ${ }^{1} \mathrm{H}$ NMR $(400 \mathrm{MHz}$, $\left.\mathrm{CDCl}_{3}\right): \delta=5.84-5.68(\mathrm{~m}, 1 \mathrm{H}), 5.18-5.04(\mathrm{~m}, 4 \mathrm{H}), 4.53(\mathrm{~s}, 2 \mathrm{H})$, 3.90-3.75 (m, $4 \mathrm{H}), 2.09$ (s, $3 \mathrm{H}), 1.45$ (s, $9 \mathrm{H}) \mathrm{ppm} .{ }^{13} \mathrm{C} \mathrm{NMR}$ $\left(100 \mathrm{MHz}, \mathrm{CDCl}_{3}\right): \delta=170.5,155.5,140.0,133.7,116.5,114.6$, 78.9, 65.0, 48.6, 48.3, 28.3, 20.8 ppm. IR (neat): $\tilde{v}=3083(\mathrm{w}), 2977$ (m), 2931 (m), 1745 (s), 1697 (s), 1456 (s), 1405 (s), 1366 (s) cm ${ }^{-1}$. LRMS (CI with $\left.\mathrm{NH}_{3}\right): m / z(\%)=270$ (16), 231 (65), 214 (29), 170 (100). HRMS (CI with $\mathrm{NH}_{3}$ ): calcd. for $\mathrm{C}_{14} \mathrm{H}_{24} \mathrm{NO}_{4}[\mathrm{M}+$ $\mathrm{H}]^{+}$270.1714; found 270.1705. $\mathrm{C}_{14} \mathrm{H}_{23} \mathrm{NO}_{4}$ (269.16): calcd. C 62.43, H 8.61, N 5.20; found C 62.63, H 8.39, N 5.15.

tert-Butyl Allyl[2-(hydroxymethyl)allyl]carbamate: tert-Butyl [2(acetoxymethyl)allyl]allylcarbamate (19) $(0.155 \mathrm{~g}, 0.57 \mathrm{mmol})$ and methanol $(2.9 \mathrm{~mL})$ were mixed to form a cloudy solution. Potassium carbonate $(0.079 \mathrm{~g}, 0.57 \mathrm{mmol})$ was dissolved in a minimal amount of $\mathrm{H}_{2} \mathrm{O}(0.25 \mathrm{~mL})$ and added to the solution. The reaction mixture was stirred for $2.5 \mathrm{~h}$ then diluted with $\mathrm{H}_{2} \mathrm{O}$ (ca. $10 \mathrm{~mL}$ ), and the methanol removed in vacuo. The mixture was extracted with three portions of $\mathrm{CH}_{2} \mathrm{Cl}_{2}$, and the combined organic extracts werer dried $\left(\mathrm{MgSO}_{4}\right)$. The solvent was removed in vacuo to yield $0.118 \mathrm{~g}(91 \%)$ of a clear colorless oil which was typically used without further purification. An analytically pure sample was obtained by chromatography (10-25\% EtOAc/hexanes gradient). $R_{f}=0.30$ (25\% EtOAc/hexanes). ${ }^{1} \mathrm{H}$ NMR (300 MHz, $\left.\mathrm{CDCl}_{3}\right): \delta=5.71-5.80$ (m, 1 H), 5.17-5.08 (m, 3 H), 4.93 (s, $1 \mathrm{H}), 4.01$ (s, $2 \mathrm{H}), 3.89$ (s, 2 $\mathrm{H}), 3.74$ (s, $2 \mathrm{H}), 3.34$ (br. s, $1 \mathrm{H}), 1.46$ (s, $9 \mathrm{H}) \mathrm{ppm} .{ }^{13} \mathrm{C} \mathrm{NMR}$ $\left(100 \mathrm{MHz}, \mathrm{CDCl}_{3}\right): \delta=156.6,144.9,133.4,116.8,113.6,80.4,63.7$, 49.0, 48.4, 28.3 ppm. IR (neat): $\tilde{v}=3442(\mathrm{~m}, \mathrm{br}), 3082(\mathrm{w}), 2977$ (w), 2929 (m), 1696 (s), 1675 (s), 1458 (s), 1410 (s), 1366 (s) cm $\mathrm{cm}^{-1}$. LRMS (EI, $70 \mathrm{eV}): m / z(\%)=227$ (0.086), 171, (29), 154 (13), 126 (10), 100 (28), 57 (100). HRMS (CI, with $\mathrm{NH}_{3}$ ): calcd. for $\mathrm{C}_{12} \mathrm{H}_{22} \mathrm{NO}_{3}[\mathrm{M}+\mathrm{H}]^{+}$228.1600; found 228.1597. $\mathrm{C}_{12} \mathrm{H}_{21} \mathrm{NO}_{3}$ (227.15): calcd. C 63.41, H 9.31, N 6.16; found C 63.49, H 9.34, N 6.07 .

tert-Butyl 3-(Hydroxymethyl)-2,5-dihydropyrrole-1-carbamate (11): tert-Butyl allyl[2-(hydroxymethyl)allyl]carbamate $(0.0564 \mathrm{~g}, 0.248$ mmol) was dissolved in $\mathrm{CH}_{2} \mathrm{Cl}_{2}(5 \mathrm{~mL})$. Grubb's catalyst (0.0102 g, $0.0124 \mathrm{mmol}, 5 \mathrm{~mol}-\%$ ) was added and the mixture refluxed for $7 \mathrm{~h}$. The reaction mixture was then cooled to room temp., opened to air, and stirred for $15 \mathrm{~h}$. The crude reaction mixture was then adsorbed onto silica gel and immediately chromatographed $(25-50 \%$ EtOAc/hexanes gradient) to give $0.0435 \mathrm{~g}(88 \%)$ of a brown oil. $R_{f}$ $=0.30(50 \%$ EtOAc/hexanes $) .{ }^{1} \mathrm{H}$ NMR $\left(400 \mathrm{MHz}, \mathrm{CDCl}_{3}\right.$, $-20^{\circ} \mathrm{C}$ ): $\delta=5.56(\mathrm{~d}, J=9.2 \mathrm{~Hz}, 1 \mathrm{H}), 4.29$ (br. s, $\left.1 \mathrm{H}\right), 4.09$ (s, 2 $\mathrm{H}), 4.02-4.00(\mathrm{~m}, 4 \mathrm{H}), 1.37$ (s, $9 \mathrm{H}) \mathrm{ppm} .{ }^{13} \mathrm{C}$ NMR $(100 \mathrm{MHz}$, $\left.\mathrm{CDCl}_{3},-20^{\circ} \mathrm{C}\right): \delta=154.4,154.4,139.5,139.3,119.6,119.5,79.6$, $79.6,59.2,59.2,53.1,53.0,52.7,52.6,28.2,28.2 \mathrm{ppm}$. IR (neat): $\tilde{v}$ $=3429$ (m, br.), $2970(\mathrm{~m}), 2860(\mathrm{~m}), 1702$ (s), 1682 (s), 1657 (s), $1412(\mathrm{~s}) \mathrm{cm}^{-1}$. LRMS (EI, $\left.70 \mathrm{eV}\right): \mathrm{m} / z(\%)=199(0.094), 184$ (0.021), 142 (25), 126 (22), 112 (19), 98 (6), 80 (15), 68 (54), 57 (100). HRMS (CI, $\left.\mathrm{NH}_{3}\right)$ : calcd. for $\mathrm{C}_{10} \mathrm{H}_{18} \mathrm{NO}_{3}[\mathrm{M}+\mathrm{H}]^{+}$ 200.1287; found 200.1288. $\mathrm{C}_{10} \mathrm{H}_{17} \mathrm{NO}_{3}$ (200.13): calcd. $\mathrm{C} 60.28, \mathrm{H}$ 8.60, N 7.03; found C 60.41, H 8.60, N 6.97.

tert-Butyl 3-(Chloromethyl)-2,5-dihydropyrrole-1-carbamate (10): (Benzylidene)dichloridobis(tricyclohexylphosphane)ruthenium $(0.286 \mathrm{~g}$, $0.35 \mathrm{mmol})$ was added to $\mathbf{1 8}(1.71 \mathrm{~g}, 6.96 \mathrm{mmol})$ in $\mathrm{CH}_{2} \mathrm{Cl}_{2}$ $(130 \mathrm{~mL})$, and the mixture was heated to reflux. After $16 \mathrm{~h}$, the reaction was not progressing so an additional portion of the catalyst $(60 \mathrm{mg}, 73 \mu \mathrm{mol})$ was added. The reaction's progression had stopped again after $24 \mathrm{~h}$, and another portion of the catalyst (60 mg, $73 \mu \mathrm{mol}$ ) was added. After a total of $3 \mathrm{~d}$, the mixture was cooled to room temp., opened to air for $24 \mathrm{~h}$, and then concentrated. The residue was chromatographed (5\% to $20 \%$ ethyl acetate/hexanes, gradient) to yield $1.34 \mathrm{~g} \mathrm{(88 \% )} \mathrm{of} \mathrm{the} \mathrm{title} \mathrm{compound.}$ $R_{f}=0.13$ ( $10 \%$ ethyl acetate/hexanes). IR (neat): $\tilde{v}=1700(\mathrm{~s}) \mathrm{cm}^{-1}$. ${ }^{1} \mathrm{H}$ NMR (400 MHz, $\mathrm{CDCl}_{3}$, containing rotamers): $\delta=5.70(\mathrm{~d}, J$ $=17.5 \mathrm{~Hz}, 1 \mathrm{H}), 4.11-4.00(\mathrm{~m}, 1 \mathrm{H}), 4.06(\mathrm{~s}, 2 \mathrm{H}), 4.04(\mathrm{~s}, 2 \mathrm{H})$, $1.38(\mathrm{~s}, 9 \mathrm{H}) \mathrm{ppm} .{ }^{13} \mathrm{C} \mathrm{NMR}\left(100 \mathrm{MHz}, \mathrm{CDCl}_{3}\right): \delta=153.96$, $135.48,135.41,124.41,124.38,79.31,79.26,52.96,52.80,40.14$, 40.08, $28.17 \mathrm{ppm}$. MS (EI, $70 \mathrm{eV}): \mathrm{m} / \mathrm{z}(\%)=57(100)[t \mathrm{Bu}]^{+}$. HRMS $\left(\mathrm{CI} / \mathrm{NH}_{3}\right)$ : calcd. for $\mathrm{C}_{10} \mathrm{H}_{16} \mathrm{ClNO}_{2}[\mathrm{M}+\mathrm{H}]^{+}$218.0948; found 218.0943 .

tert-Butyl 2-(Acetoxymethyl)-2,5-dihydropyrrole-1-carbamate (12): Acetic anhydride $(0.161 \mathrm{~mL}, 1.71 \mathrm{mmol})$ was added to tert-butyl 2(hydroxymethyl)-2,5-dihydropyrrole-1-carboxylate (11) (0.113 g, $0.57 \mathrm{mmol})$ in $\mathrm{CH}_{2} \mathrm{Cl}_{2}(12 \mathrm{~mL})$, followed by the addition of 4 -(dimethylamino)pyridine $(0.209 \mathrm{~g}, 1.71 \mathrm{mmol})$. After $16 \mathrm{~h}$, the mixture was diluted with ethyl acetate, washed (saturated $\mathrm{NaHCO}_{3}$, then brine), dried $\left(\mathrm{Na}_{2} \mathrm{SO}_{4}\right)$, and concentrated. The residue was chromatographed (10\% ethyl acetate/hexanes) to yield $0.133 \mathrm{~g}(96 \%)$ of the title compound. $R_{f}=0.12(10 \%$ ethyl acetate/hexanes). IR (neat): $\tilde{\mathrm{v}}=1745$ (s), 1698 (s) $\mathrm{cm}^{-1} \cdot{ }^{1} \mathrm{H}$ NMR (400 MHz, $\mathrm{CDCl}_{3}$, containing rotamers): $\delta=5.80(\mathrm{~s}, 0.56 \mathrm{H}), 5.73(\mathrm{~s}, 0.43 \mathrm{H}), 5.62(\mathrm{~s}$, $1 \mathrm{H}), 4.64(\mathrm{~s}, 0.43 \mathrm{H}), 4.53(\mathrm{~s}, 0.56 \mathrm{H}), 4.29(\mathrm{dd}, J=20.7,10.6 \mathrm{~Hz}$, $1 \mathrm{H}), 4.16-3.94(\mathrm{~m}, 3 \mathrm{H}), 1.94(\mathrm{~s}, 3 \mathrm{H}), 1.40(\mathrm{~s}, 9 \mathrm{H}) \mathrm{ppm} .{ }^{13} \mathrm{C}$ NMR $\left(100 \mathrm{MHz}, \mathrm{CDCl}_{3}\right): \delta=170.68,154.00,153.86,127.13$, 126.93, 79.84, 79.47, 64.34, 63.86, 63.04, 62.82, 53.57, 53.42, 28.21, 20.57, $20.56 \mathrm{ppm}$. MS (EI, $70 \mathrm{eV}): \mathrm{m} / z(\%)=168$ (44) $[\mathrm{M}-$ $\mathrm{O} t \mathrm{Bu}]^{+}, 126(44)\left[\mathrm{M}-\mathrm{C}_{2} \mathrm{H}_{2} \mathrm{O}-\mathrm{O} t \mathrm{Bu}\right]^{+}, 112(100)[\mathrm{M}+\mathrm{H}-t \mathrm{Bu}-$ $\left.\mathrm{CH}_{2} \mathrm{O}_{2} \mathrm{CCH}_{3}\right]^{+}$. HRMS (ESI): calcd. for $\mathrm{C}_{12} \mathrm{H}_{19} \mathrm{NO}_{4}[\mathrm{M}+\mathrm{Na}]^{+}$ 264.1212; found 264.1217.

tert-Butyl 1-I(Triisopropylsilyloxy)methyl|allylcarbamate (25): Imidazole $(19.8 \mathrm{mg}, 0.29 \mathrm{mmol})$ was added to tert-butyl 1-(hydroxymethyl)allylcarbamate (24) $(18.1 \mathrm{mg}, 97.0 \mu \mathrm{mol})$ and triisopropylsilyl chloride $(30 \mu \mathrm{L}, 0.14 \mathrm{mmol})$ in DMF $(0.25 \mathrm{~mL})$. After $2 \mathrm{~h}$, the mixture was diluted with $\mathrm{CH}_{2} \mathrm{Cl}_{2}$, washed with water, dried $\left(\mathrm{Na}_{2} \mathrm{SO}_{4}\right)$, and concentrated. The residue was chromatographed ( $1 \%$ to $5 \%$ ethyl acetate/hexanes, gradient) to yield $33.3 \mathrm{mg}(100 \%)$ 
of the title compound. $R_{f}=0.32$ ( $5 \%$ ethyl acetate/hexanes). IR (neat): $\tilde{v}=3354$ (br.), 1720 (s) $\mathrm{cm}^{-1} .{ }^{1} \mathrm{H} \mathrm{NMR} \mathrm{(400} \mathrm{MHz,} \mathrm{CDCl}_{3}$ ): $\delta=5.83(\mathrm{ddd}, J=17.2,10.4,5.4 \mathrm{~Hz}, 1 \mathrm{H}), 5.18(\mathrm{~d}, J=17.2 \mathrm{~Hz}, 1$ H), $5.10(\mathrm{dt}, J=10.2,1.5 \mathrm{~Hz}, 1 \mathrm{H}), 4.84(\mathrm{~s}, 1 \mathrm{H}), 4.14(\mathrm{~s}, 1 \mathrm{H})$, 3.74 (dd, $J=9.5,4.4 \mathrm{~Hz}, 1 \mathrm{H}), 3.68(\mathrm{dd}, J=9.7,4.4 \mathrm{~Hz}, 1 \mathrm{H}), 1.41$ (s, $9 \mathrm{H}), 1.04(\mathrm{~s}, 3 \mathrm{H}), 1.02(\mathrm{~s}, 18 \mathrm{H}) \mathrm{ppm} .{ }^{13} \mathrm{C} \mathrm{NMR}(100 \mathrm{MHz}$, $\left.\mathrm{CDCl}_{3}\right): \delta=155.50,136.72,115.46,79.16,65.64,54.36,28.26$, 17.80, $11.77 \mathrm{ppm}$. MS (EI, $70 \mathrm{eV}): \mathrm{m} / \mathrm{z}(\%)=270$ (8) $[\mathrm{M}-$ $\mathrm{O} t \mathrm{Bu}]^{+}, 244(100)\left[\mathrm{M}-\mathrm{O} t \mathrm{Bu}-\mathrm{C}_{2} \mathrm{H}_{2}\right]^{+}$. HRMS (ESI): calcd. for $\mathrm{C}_{18} \mathrm{H}_{37} \mathrm{NO}_{3} \mathrm{Si}[\mathrm{M}+\mathrm{Na}]^{+}$366.2440; found 366.2430.

tert-Butyl [2-(Chloromethyl)allyl]\{1-[(triisopropylsilyloxy)methyl]allyl carbamate (26): Sodium hydride $(0.296 \mathrm{~g}$ of $60 \mathrm{wt} .-\%$ suspension in mineral oil, $7.4 \mathrm{mmol})$ was added to $25(1.27 \mathrm{~g}, 3.7 \mathrm{mmol})$ and 3-chloro-2-(chloromethyl)-1-propene $(0.856 \mathrm{~mL}, 7.4 \mathrm{mmol})$ in DMF $(9 \mathrm{~mL})$. After $4 \mathrm{~h}$, water $(3 \mathrm{~mL})$, was slowly added, and the mixture was extracted with diethyl ether, washed with water, dried $\left(\mathrm{MgSO}_{4}\right)$ and concentrated. The residue was chromatographed $(1 \%$ to $5 \%$ ethyl acetate/hexanes, gradient) to yield $1.04 \mathrm{~g}(65 \%)$ of the title compound. $R_{f}=0.36$ ( $5 \%$ ethyl acetate/hexanes). IR (neat): $\tilde{v}$ $=1699(\mathrm{~s}) \mathrm{cm}^{-1} \cdot{ }^{1} \mathrm{H}$ NMR $\left(400 \mathrm{MHz}, \mathrm{CDCl}_{3}\right): \delta=5.90(\mathrm{~s}, 1 \mathrm{H})$, 5.19-5.07 (m, $4 \mathrm{H}), 4.34-4.15(\mathrm{~m}, 1 \mathrm{H}), 4.07-3.83(\mathrm{~m}, 1 \mathrm{H}), 4.05$ $(\mathrm{d}, J=13.2 \mathrm{~Hz}, 1 \mathrm{H}), 4.00(\mathrm{~s}, 2 \mathrm{H}), 3.85(\mathrm{~d}, J=16.8 \mathrm{~Hz}, 1 \mathrm{H})$, $3.76(\mathrm{dd}, J=10.1,5.5 \mathrm{~Hz}, 1 \mathrm{H}), 1.40(\mathrm{~s}, 9 \mathrm{H}), 1.02(\mathrm{~s}, 18 \mathrm{H}), 1.01$ (s, $3 \mathrm{H}) \mathrm{ppm} .{ }^{13} \mathrm{C}$ NMR $\left(100 \mathrm{MHz}, \mathrm{CDCl}_{3}\right): \delta=155.18,141.82$, $134.69,117.33,115.33,79.75,70.28,64.04,61.40,48.73,45.93$, $28.20,17.82,11.74 \mathrm{ppm}$. MS (EI, $70 \mathrm{eV}): m / z(\%)=332(100)[\mathrm{M}$ $+\mathrm{H}-t \mathrm{Bu}-i \mathrm{Pr}]^{+}, 188(58)\left[\mathrm{M}+\mathrm{H}-\mathrm{CH}_{2} \mathrm{OTips}-t \mathrm{Bu}\right]^{+}, 144(72)$ $\left[\mathrm{M}-\mathrm{CH}_{2} \mathrm{OTIPS}-\mathrm{O} t \mathrm{Bu}-\mathrm{C}_{2} \mathrm{H}_{3}\right]^{+}, 57(91)[t \mathrm{Bu}]^{+}$. HRMS (ESI): calcd. for $\mathrm{C}_{22} \mathrm{H}_{42} \mathrm{ClNO}_{3} \mathrm{Si}[\mathrm{M}+\mathrm{Na}]^{+}$454.2520; found 454.2530.

tert-Butyl [2-(Chloromethyl)allyl][1-(hydroxymethyl)allyl]carbamate (27): Tetrabutylammonium fluoride $(4.4 \mathrm{~mL}$ of a $1.0 \mathrm{M}$ solution in THF, $4.4 \mathrm{mmol})$ was added slowly to $26(0.968 \mathrm{~g}, 2.20 \mathrm{mmol})$ in THF (44 mL). After $15 \mathrm{~min}$, ethyl acetate was added, and the mixture was washed with brine. The aqueous layer was extracted with ethyl acetate, and the combined organic layers were dried $\left(\mathrm{Na}_{2} \mathrm{SO}_{4}\right)$ and concentrated. The residue was chromatographed (5\% to $30 \%$ ethyl acetate/hexanes, gradient) to yield $0.523 \mathrm{~g}(86 \%)$ of the title compound. $R_{f}=0.32$ (30\% ethyl acetate/hexanes). IR (neat): $\tilde{v}=$ 3450 (br), 1693 (s) $\mathrm{cm}^{-1} .{ }^{1} \mathrm{H}$ NMR (400 MHz, $\left.\mathrm{CDCl}_{3}\right): \delta=5.89$ (ddd, $J=17.0,10.2,6.6 \mathrm{~Hz}, 1 \mathrm{H}), 5.26(\mathrm{~s}, 2 \mathrm{H}), 5.23(\mathrm{~d}, J=$ $10.6 \mathrm{~Hz}, 1 \mathrm{H}), 5.16(\mathrm{~d}, J=17.2 \mathrm{~Hz}, 1 \mathrm{H}), 5.12(\mathrm{~s}, 1 \mathrm{H}), 4.23(\mathrm{q}, J$ $=6.2 \mathrm{~Hz}, 1 \mathrm{H}), 4.10-3.76(\mathrm{~m}, 4 \mathrm{H}), 4.04(\mathrm{~s}, 2 \mathrm{H}), 2.05(\mathrm{~s}, 1 \mathrm{H}), 1.45$ (s, $9 \mathrm{H}) \mathrm{ppm} .{ }^{13} \mathrm{C}$ NMR $\left(100 \mathrm{MHz}, \mathrm{CDCl}_{3}\right): \delta=156.16,142.08$, $133.69,117.86,115.83,80.59,63.40,61.68,51.28,48.25,45.74$, $28.11 \mathrm{ppm}$. MS (EI, $70 \mathrm{eV}): m / z(\%)=188(52)[\mathrm{M}+\mathrm{H}-t \mathrm{Bu}-$ $\left.\mathrm{CH}_{2} \mathrm{OH}\right]^{+}, 144$ (64) $\left[\mathrm{M}-\mathrm{CH}_{2} \mathrm{OH}-\mathrm{C}_{2} \mathrm{H}_{3}-\mathrm{O} t \mathrm{Bu}\right]^{+}, 57$ (100) $[t \mathrm{Bu}]^{+}$. HRMS (ESI): calcd. for $\mathrm{C}_{13} \mathrm{H}_{22} \mathrm{ClNO}_{3}[\mathrm{M}+\mathrm{Na}]^{+} 298.1186$; found 298.1190

tert-Butyl 4-(Chloromethyl)-2-(hydroxymethyl)-2,5-dihydropyrrole1-carbamate Acid (28): [1,3-Bis(2,4,6-trimethylphenyl)-2-imidazolidinylidene]dichlorido(phenylmethylene)(tricyclohexylphosphane)ruthenium (Grubbs' catalyst, 2nd generation) $(0.144 \mathrm{~g}, 0.17 \mathrm{mmol})$ was added to $27(0.937 \mathrm{~g}, 3.40 \mathrm{mmol})$ in $\mathrm{CH}_{2} \mathrm{Cl}_{2}(68 \mathrm{~mL})$, and the mixture was heated to reflux. After $10 \mathrm{~h}$, the reaction was not progressing so an additional portion of the catalyst $(62 \mathrm{mg}, 73 \mu \mathrm{mol})$ was added. After a total of $15 \mathrm{~h}$, the mixture was cooled to room temp. and then concentrated. The residue was chromatographed (20\% to $30 \%$ ethyl acetate/hexanes, gradient) to yield $0.830 \mathrm{~g}$ (98\%) of the title compound. $R_{f}=0.42(40 \%$ ethyl acetate/hexanes). IR (neat): $\tilde{v}=3409$ (br.), 1698 (s) cm ${ }^{-1} \cdot{ }^{1} \mathrm{H} \mathrm{NMR}(400 \mathrm{MHz}$, $\mathrm{CDCl}_{3}$, containing rotamers): $\delta=5.63(\mathrm{~s}, 1 \mathrm{H}), 4.74(\mathrm{~s}, 1 \mathrm{H}), 4.50$ $(\mathrm{d}, J=7.4 \mathrm{~Hz}, 1 \mathrm{H}), 4.23(\mathrm{~d}, J=15.0 \mathrm{~Hz}, 1 \mathrm{H}), 4.12(\mathrm{~d}, J=$
$15.0 \mathrm{~Hz}, 1 \mathrm{H}), 4.10$ (s, $2 \mathrm{H}), 3.74(\mathrm{dd}, J=11.4,5.1 \mathrm{~Hz}, 1 \mathrm{H}), 3.58$ (dd, $J=11.4,7.3 \mathrm{~Hz}, 1 \mathrm{H}), 1.48(\mathrm{~s}, 9 \mathrm{H}) \mathrm{ppm} .{ }^{13} \mathrm{C} \mathrm{NMR}(100 \mathrm{MHz}$, $\left.\mathrm{CDCl}_{3}\right) \delta=156.25,136.49,125.23,80.79,67.77,66.51,54.13$, 39.86, $28.25 \mathrm{ppm}$. MS (EI, $70 \mathrm{eV}): \mathrm{m} / z(\%)=57(100)[t \mathrm{Bu}]^{+}$ HRMS (ESI): calcd. for $\mathrm{C}_{11} \mathrm{H}_{18} \mathrm{ClNO}_{3}[\mathrm{M}+\mathrm{H}]^{+}$248.1053; found 248.1055

tert-Butyl 2-(Acetoxymethyl)-4-(chloromethyl)-2,5-dihydropyrrole-1carbamate (13): Acetic anhydride $(0.164 \mathrm{~mL}, 1.74 \mathrm{mmol})$ was added to $28(0.145 \mathrm{~g}, 0.580 \mathrm{mmol})$ in $\mathrm{CH}_{2} \mathrm{Cl}_{2}(12 \mathrm{~mL})$, followed by the addition of 4-(dimethylamino)pyridine $(0.212 \mathrm{~g}, 1.74 \mathrm{mmol})$. After $1.5 \mathrm{~h}$, the mixture was diluted with ethyl acetate, washed (saturated $\mathrm{NaHCO}_{3}$, then brine), dried $\left(\mathrm{Na}_{2} \mathrm{SO}_{4}\right)$, and concentrated. The residue was chromatographed (10\% to $20 \%$ ethyl acetate/hexanes, gradient) to yield $0.143 \mathrm{~g}(85 \%)$ of the title compound. $R_{f}=0.32(10 \%$ ethyl acetate/hexanes). IR (neat): $\tilde{v}=1743$ (s), $1701(\mathrm{~s}) \mathrm{cm}^{-1} \cdot{ }^{1} \mathrm{H}$ NMR (400 MHz, $\mathrm{CDCl}_{3}$, containing rotamers): $\delta=5.71(\mathrm{~d}, J=$ $5.9 \mathrm{~Hz}, 1 \mathrm{H}), 4.74(\mathrm{~s}, 0.5 \mathrm{H}), 4.62(\mathrm{~s}, 0.5 \mathrm{H}), 4.40$ (ddd, $J=23.2$, $10.8,2.9 \mathrm{~Hz}, 1 \mathrm{H}), 4.28(\mathrm{~d}, J=15.0 \mathrm{~Hz}, 1 \mathrm{H}), 4.21-4.07(\mathrm{~m}, 4 \mathrm{H})$, $2.02(\mathrm{~s}, 3 \mathrm{H}), 1.48(\mathrm{~s}, 9 \mathrm{H}) \mathrm{ppm} .{ }^{13} \mathrm{C} \mathrm{NMR}\left(100 \mathrm{MHz}, \mathrm{CDCl}_{3}\right): \delta$ $=170.74,153.87,137.17,136.93,125.80,125.59,80.23,79.91$, 78.16, 63.57, 53.69, 53.55, 39.91, 39.80, 28.26, 20.60 ppm. MS (EI, $70 \mathrm{eV}): m / z(\%)=160(72)\left[\mathrm{M}+\mathrm{H}-t \mathrm{Bu}-\mathrm{CH}_{2} \mathrm{OAc}\right]^{+}, 57(100)$ $[t \mathrm{Bu}]^{+}$. HRMS (ESI): calcd. for $\mathrm{C}_{13} \mathrm{H}_{20} \mathrm{ClNO}_{4}[\mathrm{M}+\mathrm{Na}]^{+}$312.0979; found 312.0987 .

tert-Butyl $\quad\left(2 R^{*}, 5 S^{*}\right)-2,5$-Bis(acetoxymethyl)-2,5-dihydropyrrole-1carbamate (14): The ester $2^{[13]}(0.147 \mathrm{~g}, 0.515 \mathrm{mmol})$ in THF $(1.25 \mathrm{~mL})$ was added slowly to $\mathrm{LiBH}_{4}(33.7 \mathrm{mg}, 1.54 \mathrm{mmol})$ in THF $(3.75 \mathrm{~mL})$. After $1 \mathrm{~h}, 1 \mathrm{~m} \mathrm{HCl}(4 \mathrm{~mL})$ was added dropwise, and the mixture was extracted with ethyl acetate, dried $\left(\mathrm{MgSO}_{4}\right)$, and concentrated. The residue was dissolved in $\mathrm{CH}_{2} \mathrm{Cl}_{2}(10 \mathrm{~mL})$, and acetic anhydride $(0.243 \mathrm{~mL}, 2.57 \mathrm{mmol})$ and 4-(dimethylamino)pyridine $(0.189 \mathrm{~g}, 1.54 \mathrm{mmol})$ were added in succession. After $2 \mathrm{~h}$, the mixture was diluted with ethyl acetate, washed (saturated $\mathrm{NaHCO}_{3}$, then brine), dried $\left(\mathrm{Na}_{2} \mathrm{SO}_{4}\right)$, and concentrated. The residue was chromatographed ( $20 \%$ ethyl acetate/hexanes) to yield $0.131 \mathrm{~g}(81 \%)$ of the title compound. $R_{f}=0.22(20 \%$ ethyl acetate/hexanes). IR (neat): $\tilde{v}=1743$ (s), 1700 (s) $\mathrm{cm}^{-1} \cdot{ }^{1} \mathrm{H}$ NMR $\left(400 \mathrm{MHz}, \mathrm{CDCl}_{3}\right): \delta=5.75(\mathrm{~d}, J=6.6 \mathrm{~Hz}, 1 \mathrm{H}), 5.70(\mathrm{~d}, J=$ $6.2 \mathrm{~Hz}, 1 \mathrm{H}), 4.67(\mathrm{q}, J=2.2 \mathrm{~Hz}, 1 \mathrm{H}), 4.55(\mathrm{dd}, J=5.1,4.8 \mathrm{~Hz}$, $1 \mathrm{H}), 4.47(\mathrm{dd}, J=10.8,5.1 \mathrm{~Hz}, 1 \mathrm{H}), 4.38(\mathrm{dd}, J=11.0,2.2 \mathrm{~Hz}$, $1 \mathrm{H}), 4.34(\mathrm{dd}, J=11.0,5.1 \mathrm{~Hz}, 1 \mathrm{H}), 4.20(\mathrm{dd}, J=11.0,5.1 \mathrm{~Hz}$, $1 \mathrm{H}), 2.05(\mathrm{~s}, 3 \mathrm{H}), 1.48(\mathrm{~s}, 9 \mathrm{H}) \mathrm{ppm} .{ }^{13} \mathrm{C} \mathrm{NMR}(100 \mathrm{MHz}$, $\left.\mathrm{CDCl}_{3}\right): \delta=170.89,154.17,128.65,80.57,65.20,64.72,63.75$, 63.53, 28.32, $20.79 \mathrm{ppm}$. MS (EI, $70 \mathrm{eV}): \mathrm{m} / z(\%)=124$ (94) $[\mathrm{M}-$ $\left.t \mathrm{Bu}-\mathrm{CH}_{2} \mathrm{OAc}-\mathrm{OAc}\right]^{+}, 80(100)\left[\mathrm{M}-\mathrm{Boc}-\mathrm{CH}_{2} \mathrm{OAc}-\mathrm{OAc}\right]^{+}$, 57 (52) $[t \mathrm{Bu}]^{+}$. HRMS (ESI): calcd. for $\mathrm{C}_{15} \mathrm{H}_{23} \mathrm{NO}_{6}[\mathrm{M}+\mathrm{Na}]^{+}$ 336.1423 ; found 336.1419 .

tert-Butyl $\quad\left(2 S^{*}, 5 S^{*}\right)-2,5$-Bis(acetoxymethyl)-2,5-dihydropyrrole-1carbamate (15): 1-tert-Butyl 2,5-dimethyl trans-2,5-dihydropyrrole1,2,5-tricarboxylate $(\mathbf{3 0})(106 \mathrm{mg}, 0.371 \mathrm{mmol})$ in THF $(0.5 \mathrm{~mL})$ was added slowly to super hydride $(1.86 \mathrm{~mL}$ of a $1.0 \mathrm{M}$ solution in THF, $1.86 \mathrm{mmol})$ in THF $(1.5 \mathrm{~mL})$. After $20 \mathrm{~h}, 1 \mathrm{~m} \mathrm{HCl}(1.5 \mathrm{~mL})$ was added dropwise, and the mixture was extracted with ethyl acetate, dried $\left(\mathrm{MgSO}_{4}\right)$, and concentrated. The residue was dissolved in $\mathrm{CH}_{2} \mathrm{Cl}_{2}(7.5 \mathrm{~mL})$, and acetic anhydride $(0.175 \mathrm{~mL}, 1.85 \mathrm{mmol})$ and 4-(dimethylamino)pyridine $(136 \mathrm{mg}, 1.11 \mathrm{mmol})$ were added in succession. After $1 \mathrm{~h}$, the mixture was diluted with ethyl acetate, washed (saturated $\mathrm{NaHCO}_{3}$, then brine), dried $\left(\mathrm{Na}_{2} \mathrm{SO}_{4}\right)$, and concentrated. The residue was chromatographed $(20 \%$ ethyl acetate/ hexanes) to yield $95.0 \mathrm{mg}(82 \%)$ of the title compound. $R_{f}=0.20$ (20\% ethyl acetate/hexanes). IR (neat): $\tilde{v}=1743(\mathrm{~s}), 1698(\mathrm{~s}) \mathrm{cm}^{-1}$. ${ }^{1} \mathrm{H}$ NMR $\left(400 \mathrm{MHz}, \mathrm{CDCl}_{3}\right): \delta=5.75(\mathrm{~d}, J=6.6 \mathrm{~Hz}, 1 \mathrm{H}), 5.70$ 
$(\mathrm{d}, J=6.2 \mathrm{~Hz}, 1 \mathrm{H}), 4.67(\mathrm{q}, J=2.2 \mathrm{~Hz}, 1 \mathrm{H}), 4.55(\mathrm{dd}, J=6.8$, $5.1 \mathrm{~Hz}, 1 \mathrm{H}), 4.47(\mathrm{dd}, J=10.8,2.6 \mathrm{~Hz}, 1 \mathrm{H}), 4.39$ (dd, $J=11.0$, $2.2 \mathrm{~Hz}, 1 \mathrm{H}), 4.34(\mathrm{dd}, J=11.0,5.1 \mathrm{~Hz}, 1 \mathrm{H}), 4.20(\mathrm{dd}, J=11.0$, $5.1 \mathrm{~Hz}, 1 \mathrm{H}), 1.99$ (s, $3 \mathrm{H}), 1.45$ (s, $9 \mathrm{H}) \mathrm{ppm} .{ }^{13} \mathrm{C} \mathrm{NMR}(100 \mathrm{MHz}$, $\left.\mathrm{CDCl}_{3}\right): \delta=170.68,153.21,128.56,128.42,80.46,65.19,63.71$, 63.57, 62.65, 28.29, $20.61 \mathrm{ppm}$. MS (EI, $70 \mathrm{eV}): \mathrm{m} / z(\%)=124(95)$ $\left[\mathrm{M}-t \mathrm{Bu}-\mathrm{CH}_{2} \mathrm{OAc}-\mathrm{OAc}\right]^{+}, 80(100)\left[\mathrm{M}-\mathrm{Boc}-\mathrm{CH}_{2} \mathrm{OAc}-\right.$ $\mathrm{OAc}^{+}, 57$ (56) $[t \mathrm{Bu}]^{+}$. HRMS (ESI): calcd. for $\mathrm{C}_{15} \mathrm{H}_{23} \mathrm{NO}_{6}[\mathrm{M}+$ $\mathrm{Na}]^{+}$336.1423; found 336.1423 .

2-[3-(Hydroxymethyl)-2,5-dihydropyrrol-1-yl]-1,4-naphthoquinone (34): tert-Butyl 3-(hydroxymethyl)-2,5-dihydropyrrole-1-carboxylate $(\mathbf{8})(0.1197 \mathrm{~g}, 0.6013 \mathrm{mmol})$ was dissolved in $\mathrm{CH}_{2} \mathrm{Cl}_{2}(1.4 \mathrm{~mL})$, and trifloroacetic acid $(0.46 \mathrm{~mL}, 6.013 \mathrm{mmol})$ was added dropwise. The reaction mixture was stirred for $1 \mathrm{~h}$, at which time the solvent and excess trifluoroacetic acid were removed in vacuo to leave the trifloroacetic acid salt of the free amine which was used without purification. The crude amine salt was neutralized by the addition of a $0.2 \mathrm{M}$ solution of $\mathrm{NH}_{4} \mathrm{OH}$ in methanol ( $3.1 \mathrm{~mL}, 0.62 \mathrm{mmol}$ ). A second solution containing naphthoquinone $(0.0951 \mathrm{~g}$, $0.6013 \mathrm{mmol})$ and copper(I) bromide $(0.0086 \mathrm{~g}, 0.06013 \mathrm{mmol})$ in diethyl ether $(3 \mathrm{~mL})$ was prepared, and the flask wrapped in aluminum foil to protect it from ambient light. In a dark fume hood, the amine solution was added dropwise to the solution of the quinone. Upon addition of the first few drops, the solution turned from a deep gold color to red. The reaction mixture, open to air, was stirred for $15 \mathrm{~h}$, then the solvent removed in vacuo. The residue was taken up in water and $\mathrm{CH}_{2} \mathrm{Cl}_{2}$, the layers were separated, the organic layer was extracted with 3 portions of $\mathrm{CH}_{2} \mathrm{Cl}_{2}$. The combined organic extracts were dried $\left(\mathrm{MgSO}_{4}\right)$, and the solvent was removed in vacuo to yield an orange solid. Chromatography (50$100 \%$ EtOAc/hexanes gradient) afforded $0.0930 \mathrm{~g}$ of an orange crystalline solid. $R_{f}=0.23$ (EtOAc). M.p. $163-164{ }^{\circ} \mathrm{C} .{ }^{1} \mathrm{H}$ NMR (400 MHz, $\mathrm{CDCl}_{3}, 3$ drops of $\mathrm{CD}_{3} \mathrm{OD}$ ): $\delta=8.04$ (dd, $J=7.6$, $1.2 \mathrm{~Hz}, 1 \mathrm{H}), 8.01(\mathrm{dd}, J=7.6,1.2 \mathrm{~Hz}, 1 \mathrm{H}), 7.71(\mathrm{dd}, J=7.6$, $1.2 \mathrm{~Hz}, 1 \mathrm{H}$ ), 7.62 (dd, $J=7.6,1.2 \mathrm{~Hz}, 1 \mathrm{H}), 5.83$ (s, $1 \mathrm{H}), 5.69$ (s, $1 \mathrm{H}), 4.78$ (s, $2 \mathrm{H}), 4.28$ (s, $2 \mathrm{H}), 4.21$ (br. s, $2 \mathrm{H}$ ) ppm. ${ }^{13} \mathrm{C}$ NMR $\left(100 \mathrm{MHz}, \mathrm{CDCl}_{3}\right): \delta=183.0,182.7,148.0,134.2,133.1,131.9$, $131.7,126.5,125.6,105.1,59.7,58.5-57.5$ (br., 2 C) ppm. As further proof of structure, a low-temperature NMR spectrum was recorded to "freeze out" the vinylogous amide rotamers: ${ }^{1} \mathrm{H}$ NMR (400 MHz, $\mathrm{CDCl}_{3}, 3$ drops of $\left.\mathrm{CD}_{3} \mathrm{OD},-20^{\circ} \mathrm{C}\right): \delta=8.00-8.03(\mathrm{~m}$, $2 \mathrm{H}), 7.74(\mathrm{~d}, J=7.5 \mathrm{~Hz}, 1 \mathrm{H}), 7.65(\mathrm{~d}, J=7.5 \mathrm{~Hz}, 1 \mathrm{H}), 5.84(\mathrm{~d}$, $J=20 \mathrm{~Hz}, 1 \mathrm{H}), 5.67(\mathrm{~s}, 1 \mathrm{H}), 4.78(\mathrm{~d}, J=14.4 \mathrm{~Hz}, 2 \mathrm{H}), 4.30$ $4.19(\mathrm{~m}, 5 \mathrm{H}) \mathrm{ppm} .{ }^{13} \mathrm{C} \mathrm{NMR}\left(100 \mathrm{MHz}, \mathrm{CDCl}_{3},-20^{\circ} \mathrm{C}\right): \delta=$ 183.0, 182.8, 182.3, 147.9, 147.8, 141.0, 136.7, 134.3, 132.7, 132.01, $132.0,131.1,126.5,126.5,125.2,121.2,116.6,104.0,103.8,58.8$, 58.6, 58.4, 58.0, 57.5, 57.2 ppm. IR (neat): $\tilde{v}=3364$ (m, br.), 2918 (m), 2849 (m), $1672(\mathrm{~m}), 1608$ (m), 1589 (s), 1539 (s), 1454 (m), $1420(\mathrm{~m}) \mathrm{cm}^{-1}$. UV $\left(\mathrm{CHCl}_{3}\right): \lambda_{\max }=247,277,464,642,729 \mathrm{~nm}$ $(\log \varepsilon=3.91,3.99,3.53,3.29,3.21)$. LRMS $\left(\mathrm{CI}, \mathrm{NH}_{3}\right): \mathrm{m} / z(\%)=$ 256 (100), 238 (38), 225 (21). HRMS (CI, $\mathrm{NH}_{3}$ ): calcd. for $\mathrm{C}_{15} \mathrm{H}_{14} \mathrm{NO}_{3}[\mathrm{M}+\mathrm{H}]^{+}$256.0974; found 256.0961. $\mathrm{C}_{15} \mathrm{H}_{13} \mathrm{NO}_{3}$ (255.09): calcd. C 70.58, H 5.13, N 5.49; found C 70.61, H 5.03, N 5.27 .

2-[3-(Acetoxyoxymethyl)-2,5-dihydropyrrol-1-yl]-1,4-naphthoquinone (35): 2-[3-(Hydroxymethyl)-2,5-dihydropyrrol-1-yl]-1,4-naphthoquinone (34) $(0.0700 \mathrm{~g}, 0.275 \mathrm{mmol})$ was dissolved in pyridine $(1 \mathrm{~mL})$ in a flask wrapped in aluminum foil (to protect the contents from light). The solution was cooled in an ice bath, and acetic anhydride $(0.2800 \mathrm{~g}, 2.77 \mathrm{mmol})$ was added. After $5 \mathrm{~min}$, the bath was removed and stirring continued for $4 \mathrm{~h}$. The reaction mixture was then diluted with EtOAc (ca. $10 \mathrm{~mL}$ ) and aqueous $\mathrm{NaOH}$ ( $10 \mathrm{~mL}$ of a $15 \% \mathrm{w} / \mathrm{v}$ solution). The layers were then separated, and the organic layer was washed with $\mathrm{NaOH}$ solution $(20 \mathrm{~mL})$, followed by $\mathrm{H}_{2} \mathrm{O}(2 \times 20 \mathrm{~mL})$. The extract was dried $\left(\mathrm{MgSO}_{4}\right)$ and the solvent removed in vacuo. Chromatography $(0-30 \%$ EtOAc/ hexanes gradient) afforded $0.0817 \mathrm{~g}$ (quantitative yield) of a red solid. $R_{f}=0.045(20 \%$ EtOAc/hexanes $)$. M.p. $119.5-121{ }^{\circ} \mathrm{C} .{ }^{1} \mathrm{H}$ NMR (400 MHz, $\left.\mathrm{CDCl}_{3}\right): \delta=8.07(\mathrm{dd}, J=7.8,1.2 \mathrm{~Hz}, 1 \mathrm{H}), 8.04$ $(\mathrm{dd}, J=7.8,1.2 \mathrm{~Hz}, 1 \mathrm{H}), 7.71(\mathrm{dt}, J=7.8,1.2 \mathrm{~Hz}, 1 \mathrm{H}), 7.62(\mathrm{dt}$, $J=7.8,1.2 \mathrm{~Hz}, 1 \mathrm{H}), 5.91(\mathrm{~s}, 1 \mathrm{H}), 5.73(\mathrm{~s}, 1 \mathrm{H}), 5.17-4.58(\mathrm{~m}, 4$ $\mathrm{H}), 4.19$ (br. s, $2 \mathrm{H}$ ), 2.11 (s, $3 \mathrm{H})$ ppm. ${ }^{13} \mathrm{C} \mathrm{NMR} \mathrm{(100} \mathrm{MHz,}$ $\left.\mathrm{CDCl}_{3}\right): \delta=182.7,182.5,170.5,147.6,136.2,134.1,133.0,131.9$, 131.6, 126.4, 125.5, 120.7, 105.3, 60.5, 57.8 (br., 2 C), 20.7 ppm. IR (neat): $\tilde{v}=3067(w), 2919(w), 2848$ (w), 1738 (s), 1673 (s), 1622 (s), 1593 (s), 1565 (s), 1267 (s) $\mathrm{cm}^{-1}$. UV $\left(\mathrm{CHCl}_{3}\right): \lambda_{\max }=246,276$, 464, $642 \mathrm{~nm}(\log \varepsilon=4.04,4.15,3.56,2.81)$. LRMS (CI, $\left.\mathrm{NH}_{3}\right): m / z$ $(\%)=298$ (24), 240 (100), 224 (21), 82 (19). HRMS (CI, NH $\mathrm{NH}_{3}$ : calcd. for $\mathrm{C}_{17} \mathrm{H}_{16} \mathrm{NO}_{4}[\mathrm{M}+\mathrm{H}]^{+}$298.1079; found 298.1073 . $\mathrm{C}_{17} \mathrm{H}_{15} \mathrm{NO}_{4}$ (297.10): calcd. C 68.66, H 5.09, N 4.71; found $\mathrm{C}$ 68.67, H 5.07, N 4.47.

2-[3-(Chloromethyl)-2,5-dihydropyrrol-1-yl]-1,4-naphthoquinone (36): Trifluoroacetic acid $(0.619 \mathrm{~mL}, 8.0 \mathrm{mmol})$ was added to $\mathbf{1 0}$ (175 mg, $0.800 \mathrm{mmol}$ ) in $\mathrm{CH}_{2} \mathrm{Cl}_{2}(8 \mathrm{~mL})$. After $2 \mathrm{~h}$, the mixture was concentrated and exposed to high vacuum for $1 \mathrm{~h}$. The residue was dissolved in THF $(8 \mathrm{~mL})$, and triethylamine $(123 \mu \mathrm{L}, 0.88 \mathrm{mmol})$ was added. After $10 \mathrm{~min}$, this mixture was added to 1,4-naphthoquinone (126 mg, $0.800 \mathrm{mmol}$ ) and copper(I) bromide (23.0 mg, $0.160 \mathrm{mmol})$ in THF $(4 \mathrm{~mL})$ in the dark. The mixture was heated to reflux open to air in the dark. After $16 \mathrm{~h}$, it was cooled to room temp. and concentrated. The residue was chromatographed $(20 \%$ to $40 \%$ ethyl acetate/hexanes, gradient) to yield $111 \mathrm{mg}(51 \%)$ of the title compound. $R_{f}=0.11$ (25\% ethyl acetate/hexanes). IR (neat): $\tilde{v}=1668$ (s), 1617 (s), $1591(\mathrm{~m}), 1557$ (s) $\mathrm{cm}^{-1} \cdot{ }^{1} \mathrm{H}$ NMR $\left(500 \mathrm{MHz}, \mathrm{CDCl}_{3}\right): \delta=8.05(\mathrm{dd}, J=7.6,0.7 \mathrm{~Hz}, 1 \mathrm{H}), 8.02(\mathrm{dd}$, $J=7.7,0.7 \mathrm{~Hz}, 1 \mathrm{H}), 7.70(\mathrm{td}, J=7.6,1.5 \mathrm{~Hz}, 1 \mathrm{H}), 7.61(\mathrm{td}, J=$ 7.7, $1.5 \mathrm{~Hz}, 1 \mathrm{H}), 5.96$ (s, $1 \mathrm{H}), 5.69$ (s, $1 \mathrm{H}), 4.81$ (s, $2 \mathrm{H}), 4.22$ (s, 4 H) ppm. ${ }^{13} \mathrm{C}$ NMR $\left(100 \mathrm{MHz}, \mathrm{CDCl}_{3}\right): \delta=182.81,182.63,147.86$, 134.27, 132.99, 132.01, 131.58, 126.49, 125.58, 105.33, 58.11, $39.85 \mathrm{ppm}$. MS (EI, $70 \mathrm{eV}): m / z(\%)=273(38)[\mathrm{M}]^{+}, 238(100)$ $[\mathrm{M}-\mathrm{Cl}]^{+}, 224$ (42) $\left[\mathrm{M}-\mathrm{H}-\mathrm{CH}_{2} \mathrm{Cl}\right]^{+}$. HRMS (EI, $70 \mathrm{eV}$ ): calcd. for $\mathrm{C}_{15} \mathrm{H}_{12} \mathrm{ClNO}_{2}[\mathrm{M}]^{+}$273.0557; found 273.0556.

2-[2-(Acetoxymethyl)-2,5-dihydropyrrol-1-yl]-1,4-naphthoquinone (38): Trifluoroacetic acid $(123 \mu \mathrm{L}, 1.6 \mathrm{mmol})$ was added to 12 (39.7 mg, $0.160 \mathrm{mmol})$ in $\mathrm{CH}_{2} \mathrm{Cl}_{2}(2 \mathrm{~mL})$. After $2 \mathrm{~h}$, the mixture was concentrated and exposed to high vacuum for $1 \mathrm{~h}$. The residue was dissolved in THF $(2 \mathrm{~mL})$, and triethylamine $(24.5 \mu \mathrm{L}$, $0.176 \mathrm{mmol}$ ) was added. After $10 \mathrm{~min}$, this mixture was added to 1,4-naphthoquinone $(25.3 \mathrm{mg}, 0.160 \mathrm{mmol})$ and copper(I) bromide $(4.6 \mathrm{mg}, 32 \mu \mathrm{mol})$ in THF $(1 \mathrm{~mL})$ in the dark. The mixture was heated to reflux open to air in the dark. After $2 \mathrm{~d}$, it was cooled to room temp. and concentrated. The residue was chromatographed (10\% to $40 \%$ ethyl acetate/hexanes, gradient) to yield $23.6 \mathrm{mg}$ $(50 \%)$ of the title compound. $R_{f}=0.05(30 \%$ ethyl acetate/hexanes). IR (neat): $\tilde{v}=1741$ (s), $1674(\mathrm{~m}), 1626(\mathrm{~m}), 1592(\mathrm{~m}), 1557$ (s) $\mathrm{cm}^{-1} .{ }^{1} \mathrm{H} \mathrm{NMR}\left(400 \mathrm{MHz}, \mathrm{CDCl}_{3}\right): \delta=8.03$ (dd, $J=7.5$, $1.1 \mathrm{~Hz}, 1 \mathrm{H}), 8.00(\mathrm{dd}, J=7.7,1.1 \mathrm{~Hz}, 1 \mathrm{H}), 7.68(\mathrm{td}, J=7.5$, $1.5 \mathrm{~Hz}, 1 \mathrm{H}), 7.60(\mathrm{td}, J=7.5,1.5 \mathrm{~Hz}, 1 \mathrm{H}), 6.02(\mathrm{dd}, J=6.6$, $1.8 \mathrm{~Hz}, 1 \mathrm{H}), 5.88(\mathrm{~s}, 1 \mathrm{H}), 5.88-5.85(\mathrm{~m}, 1 \mathrm{H}), 5.51(\mathrm{~s}, 1 \mathrm{H}), 4.40$ (s, 1 H), 4.32-4.17 (m, 1 H), $4.31(\mathrm{dd}, J=11.5,2.9 \mathrm{~Hz}, 1 \mathrm{H}), 4.19$ (dd, $J=11.7,4.4 \mathrm{~Hz}, 1 \mathrm{H}), 1.97$ (s, $3 \mathrm{H}) \mathrm{ppm} .{ }^{13} \mathrm{C} \mathrm{NMR}(100 \mathrm{MHz}$, $\left.\mathrm{CDCl}_{3}\right): \delta=183.39,182.95,170.74,148.58,134.35,132.58,131.80$, $127.43,126.73,125.66,106.53,65.51,63.62,58.06,20.62 \mathrm{ppm} . \mathrm{MS}$ $(\mathrm{EI}, 70 \mathrm{eV}): m / z(\%)=297(6)[\mathrm{M}]^{+}, 237(36)[\mathrm{M}-\mathrm{HOAc}]^{+}, 224$ (100) $\left[\mathrm{M}-\mathrm{CH}_{2} \mathrm{OAc}\right]^{+}$. HRMS (EI, $70 \mathrm{eV}$ ): calcd. for $\mathrm{C}_{17} \mathrm{H}_{15} \mathrm{NO}_{4}$ $[\mathrm{M}]^{+}$297.1001; found 297.1006. 
2-[2-(Acetoxymethyl)-4-(chloromethyl)-2,5-dihydropyrrol-1-yl]-1,4naphthoquinone (39): Trifluoroacetic acid $(0.185 \mathrm{~mL}, 2.40 \mathrm{mmol})$ was added to $13(69.5 \mathrm{mg}, 0.240 \mathrm{mmol})$ in $\mathrm{CH}_{2} \mathrm{Cl}_{2}(2.5 \mathrm{~mL})$. After $3 \mathrm{~h}$, the mixture was concentrated and exposed to high vacuum for $1 \mathrm{~h}$. The residue was dissolved in THF $(2.5 \mathrm{~mL})$, and triethylamine ( $36.8 \mu \mathrm{L}, 0.264 \mathrm{mmol}$ ) was added. After $10 \mathrm{~min}$, this mixture was added to 1,4-naphthoquinone (37.9 $\mathrm{mg}, 0.240 \mathrm{mmol}$ ) and copper(I) bromide $(6.9 \mathrm{mg}, 48 \mu \mathrm{mol})$ in THF $(1 \mathrm{~mL})$ in the dark. The mixture was heated to reflux open to air in the dark. After $16 \mathrm{~h}$, it was cooled to room temp. and concentrated. The residue was chromatographed ( $30 \%$ ethyl acetate/hexanes) to yield $28.2 \mathrm{mg}$ (34\%) of the title compound. $R_{f}=0.25(50 \%$ ethyl acetate/hexanes). IR (neat): $\tilde{v}=1739(\mathrm{~s}), 1670(\mathrm{~s}), 1626(\mathrm{~m}), 1592(\mathrm{~m}), 1556(\mathrm{~s}) \mathrm{cm}^{-1} \cdot{ }^{1} \mathrm{H}$ NMR $\left(300 \mathrm{MHz}, \mathrm{CDCl}_{3}\right): \delta=8.05(\mathrm{dd}, J=8.4,1.5 \mathrm{~Hz}, 1 \mathrm{H}), 8.02$ (dd, $J=7.6,1.1 \mathrm{~Hz}, 1 \mathrm{H}), 7.71(\mathrm{td}, J=7.6,1.4 \mathrm{~Hz}, 1 \mathrm{H}), 7.63(\mathrm{td}, J=$ 7.6, $1.4 \mathrm{~Hz}, 1 \mathrm{H}), 5.89(\mathrm{~s}, 1 \mathrm{H}), 5.87(\mathrm{~s}, 1 \mathrm{H}), 5.52(\mathrm{~s}, 1 \mathrm{H}), 4.52(\mathrm{~s}$, $1 \mathrm{H}), 4.38(\mathrm{dd}, J=11.5,2.7 \mathrm{~Hz}, 1 \mathrm{H}), 4.32-4.23(\mathrm{~m}, 1 \mathrm{H}), 4.23$ (s, $2 \mathrm{H}), 4.20$ (dd, $J=11.7,4.1 \mathrm{~Hz}, 1 \mathrm{H}), 2.00$ (s, $3 \mathrm{H}) \mathrm{ppm} .{ }^{13} \mathrm{C} \mathrm{NMR}$ $\left(100 \mathrm{MHz}, \mathrm{CDCl}_{3}\right): \delta=182.98,179.85,170.56,147.86,134.26$, $132.53,132.23,131.76,126.57,125.57,107.25,96.1894 .50,84.63$, 65.96, 58.08, 39.56, $20.70 \mathrm{ppm}$. MS (EI, $70 \mathrm{eV}): \mathrm{m} / \mathrm{z}(\%)=117(97)$, 49 (100). HRMS (ESI): calcd. for $\mathrm{C}_{18} \mathrm{H}_{16} \mathrm{ClNO}_{4}[\mathrm{M}+\mathrm{Na}]^{+}$ 368.0666; found 368.0663 .

2-[cis-2,5-Bis(acetoxymethyl)-2,5-dihydropyrrol-1-yl]-1,4-naphthoquinone (40): Trifluoroacetic acid $(92 \mu \mathrm{L}, 1.2 \mathrm{mmol})$ was added to $14(39.0 \mathrm{mg}, 0.120 \mathrm{mmol})$ in $\mathrm{CH}_{2} \mathrm{Cl}_{2}(1.5 \mathrm{~mL})$. After $2.5 \mathrm{~h}$, the mixture was concentrated and exposed to high vacuum for $1 \mathrm{~h}$. The residue was dissolved in THF $(1.5 \mathrm{~mL})$, and triethylamine $(19.5 \mu \mathrm{L}$, $0.140 \mathrm{mmol}$ ) was added. After $10 \mathrm{~min}$, this mixture was added to 1,4-naphthoquinone (19.0 mg, $0.120 \mathrm{mmol}$ ) and copper(I) bromide $(3.4 \mathrm{mg}, 24 \mu \mathrm{mol})$ in THF $(1 \mathrm{~mL})$ in the dark. The mixture was heated to reflux open to air in the dark. After $3 \mathrm{~d}$, it was cooled to room temp. and concentrated. The residue was chromatographed ( $10 \%$ to $40 \%$ ethyl acetate/hexanes, gradient) to yield $23.3 \mathrm{mg}$ $(52 \%)$ of the title compound. $R_{f}=0.10(30 \%$ ethyl acetate/hexanes). IR (neat): $\tilde{v}=1741(\mathrm{~s}), 1674(\mathrm{~s}), 1631(\mathrm{~m}), 1592(\mathrm{~m}), 1550$ (s) $\mathrm{cm}^{-1} .{ }^{1} \mathrm{H}$ NMR $\left(500 \mathrm{MHz}, \mathrm{CDCl}_{3}\right): \delta=8.04(\mathrm{td}, J=7.6,1.0 \mathrm{~Hz}$, $2 \mathrm{H}), 7.72(\mathrm{td}, J=7.6,1.5 \mathrm{~Hz}, 1 \mathrm{H}), 7.64(\mathrm{td}, J=7.6,1.5 \mathrm{~Hz}, 1$ H), 6.09 (s, $1 \mathrm{H}), 6.00(\mathrm{~s}, 2 \mathrm{H}), 5.19(\mathrm{dd}, J=5.2,3.7 \mathrm{~Hz}, 2 \mathrm{H}), 4.47$ $(\mathrm{dd}, J=11.3,3.7 \mathrm{~Hz}, 2 \mathrm{H}), 4.24(\mathrm{dd}, J=11.2,5.9 \mathrm{~Hz}, 2 \mathrm{H}), 2.11$ (s, $6 \mathrm{H}) \mathrm{ppm} .{ }^{13} \mathrm{C} \mathrm{NMR}\left(100 \mathrm{MHz}, \mathrm{CDCl}_{3}\right): \delta=183.41,170.70$, 148.00, 134.27, 132.37, 132.14, 128.42, 126.76, 125.44, 107.29, $66.56,64.39,20.77 \mathrm{ppm}$. MS (EI, $70 \mathrm{eV}): \mathrm{m} / z(\%)=236(100)[\mathrm{M}-$ $\left.\mathrm{HOAc}-\mathrm{CH}_{2} \mathrm{OAc}\right]^{+}$. HRMS (ESI): calcd. for $\mathrm{C}_{20} \mathrm{H}_{19} \mathrm{NO}_{6}[\mathrm{M}+$ $\mathrm{Na}]^{+}$392.1110; found 392.1100.

2-(2-Acetoxymethyl-2,5-dihydropyrrol-1-yl)-8-hydroxy-1,4-naphthoquinone (42): Trifluoroacetic acid $(97 \mu \mathrm{L}, 1.3 \mathrm{mmol})$ was added to 12 (30.5 mg, $0.126 \mathrm{mmol})$ in $\mathrm{CH}_{2} \mathrm{Cl}_{2}(2 \mathrm{~mL})$. After $1.5 \mathrm{~h}$, the mixture was concentrated and exposed to high vacuum for $1 \mathrm{~h}$. The residue was dissolved in THF $(2 \mathrm{~mL})$, and triethylamine $(19.3 \mu \mathrm{L}$, $0.139 \mathrm{mmol}$ ) was added. After $10 \mathrm{~min}$, this mixture was added to juglone (21.9 mg, $0.126 \mathrm{mmol})$ and copper(I) bromide $(3.6 \mathrm{mg}$, $25 \mu \mathrm{mol})$ in THF $(1 \mathrm{~mL})$ in the dark. The mixture was heated to reflux open to air in the dark. After $2 \mathrm{~d}$, it was cooled to room temp. and concentrated. The residue was chromatographed $(10 \%$ to $40 \%$ ethyl acetate/hexanes, gradient) to yield $17.7 \mathrm{mg} \mathrm{(45 \% )} \mathrm{of}$ the title compound. $R_{f}=0.09$ (40\% ethyl acetate/hexanes). IR (neat): $\tilde{v}=1740$ (s), 1625 (s), 1557 (s) cm ${ }^{-1} .{ }^{1} \mathrm{H}$ NMR $(400 \mathrm{MHz}$, $\left.\mathrm{CDCl}_{3}\right): \delta=11.84(\mathrm{~s}, 1 \mathrm{H}), 7.56(\mathrm{~d}, J=5.1 \mathrm{~Hz}, 1 \mathrm{H}), 7.55(\mathrm{~d}, J=$ $4.0 \mathrm{~Hz}, 1 \mathrm{H}), 7.12(\mathrm{t}, J=4.8 \mathrm{~Hz}, 1 \mathrm{H}), 6.01(\mathrm{dd}, J=6.6,1.8 \mathrm{~Hz}, 1$ H), $5.85(\mathrm{dd}, J=6.6,2.2 \mathrm{~Hz}, 1 \mathrm{H}), 5.79(\mathrm{~s}, 1 \mathrm{H}), 5.47(\mathrm{~s}, 1 \mathrm{H}), 4.38$ (s, $1 \mathrm{H}), 4.28(\mathrm{dd}, J=11.5,3.3 \mathrm{~Hz}, 1 \mathrm{H}), 4.23-4.18(\mathrm{~m}, 1 \mathrm{H}), 4.19$ (dd, $J=11.4,4.4 \mathrm{~Hz}, 1 \mathrm{H}), 1.96$ (s, $3 \mathrm{H}) \mathrm{ppm} .{ }^{13} \mathrm{C} \mathrm{NMR}(100 \mathrm{MHz}$,
$\left.\mathrm{CDCl}_{3}\right): \delta=188.06,182.16,170.55,161.88,147.56,137.13,132.77$, $127.40,126.07,122.70,118.03,115.02,107.98,65.58,63.97,58.26$, 29.67, $20.69 \mathrm{ppm}$. MS (EI, $70 \mathrm{eV}): \mathrm{m} / z(\%)=253(46)[\mathrm{M}-$ HOAc $]^{+}, 57$ (100). HRMS (ESI): calcd. for $\mathrm{C}_{17} \mathrm{H}_{15} \mathrm{NO}_{5}[\mathrm{M}+$ $\mathrm{Na}]^{+}$336.0848; found 336.0849.

7-(2-Acetoxymethyl-2,5-dihydropyrrol-1-yl)isoquinoline-5,8-dione (44): Trifluoroacetic acid $(0.11 \mathrm{~mL}, 1.4 \mathrm{mmol})$ was added to 12 (33.8 $\mathrm{mg}, 0.140 \mathrm{mmol})$ in $\mathrm{CH}_{2} \mathrm{Cl}_{2}(2 \mathrm{~mL})$. After $1 \mathrm{~h}$, the mixture was concentrated and exposed to high vacuum for $1 \mathrm{~h}$. The residue was dissolved in THF $(2.5 \mathrm{~mL})$, and triethylamine $(22 \mu \mathrm{L}$, $0.15 \mathrm{mmol}$ ) was added. After $10 \mathrm{~min}$, this mixture was added to isoquinoline-5,8-dione $(22.3 \mathrm{mg}, 0.140 \mathrm{mmol})$ and copper(I) bromide $(4.0 \mathrm{mg}, 28 \mu \mathrm{mol})$ in THF $(1 \mathrm{~mL})$ in the dark. The mixture was heated to reflux open to air in the dark. After $16 \mathrm{~h}$, it was cooled to room temp. and concentrated. The residue was chromatographed (30\% to $60 \%$ ethyl acetate/hexanes, gradient) to yield $21.4 \mathrm{mg}(51 \%)$ of the title compound. $R_{f}=0.13(60 \%$ ethyl acetate/ hexanes). IR (neat): $\tilde{v}=1740$ (s), $1679(\mathrm{~m}), 1627$ (s), $1586(\mathrm{~m}), 1548$ (s) $\mathrm{cm}^{-1} .{ }^{1} \mathrm{H} \mathrm{NMR}\left(400 \mathrm{MHz}, \mathrm{CDCl}_{3}\right): \delta=9.25(\mathrm{~s}, 1 \mathrm{H}), 8.98(\mathrm{~d}$, $J=4.8 \mathrm{~Hz}, 1 \mathrm{H}), 7.86(\mathrm{~d}, J=5.1 \mathrm{~Hz}, 1 \mathrm{H}), 6.05(\mathrm{dd}, J=6.6$, $1.8 \mathrm{~Hz}, 1 \mathrm{H}), 5.92-5.88(\mathrm{~m}, 1 \mathrm{H}), 5.91(\mathrm{~s}, 1 \mathrm{H}), 5.52(\mathrm{~s}, 1 \mathrm{H}), 4.40$ $(\mathrm{s}, 1 \mathrm{H}), 4.33-4.24(\mathrm{~m}, 3 \mathrm{H}), 1.99(\mathrm{~s}, 3 \mathrm{H}) \mathrm{ppm} .{ }^{13} \mathrm{C} \mathrm{NMR}$ $\left(100 \mathrm{MHz}, \mathrm{CDCl}_{3}\right): \delta=182.49,180.94,170.49,155.53,153.96$, $148.28,147.98,147.83,138.15,125.46,118.09,107.56,65.61,58.07$, 29.66, 20.69 ppm. MS (EI, $70 \mathrm{eV}): \mathrm{m} / z(\%)=298(3)[\mathrm{M}]^{+}, 238(45)$ $[\mathrm{M}-\mathrm{HOAc}]^{+}, 225(100)\left[\mathrm{M}-\mathrm{CH}_{2} \mathrm{OAc}\right]^{+}$. HRMS (EI, $\left.70 \mathrm{eV}\right)$ : calcd. for $\mathrm{C}_{16} \mathrm{H}_{14} \mathrm{~N}_{2} \mathrm{O}_{4}[\mathrm{M}]^{+}$298.0954; found 298.0965.

7-[3-(Chloromethyl)-2,5-dihydropyrrol-1-yl]isoquinoline-5,8-dione (45): Trifluoroacetic acid $(0.41 \mathrm{~mL}, 5.3 \mathrm{mmol})$ was added to $\mathbf{1 0}$ (106 mg, $0.530 \mathrm{mmol})$ in $\mathrm{CH}_{2} \mathrm{Cl}_{2}(7.5 \mathrm{~mL})$. After $2 \mathrm{~h}$, the mixture was concentrated and exposed to high vacuum for $1 \mathrm{~h}$. The residue was dissolved in THF $(7.5 \mathrm{~mL})$, and triethylamine $(81 \mu \mathrm{L}$, $0.58 \mathrm{mmol}$ ) was added. After $10 \mathrm{~min}$, this mixture was added to isoquinoline-5,8-dione $(84.3 \mathrm{mg}, 0.530 \mathrm{mmol})$ and copper(I) bromide $(15.2 \mathrm{mg}, 106 \mu \mathrm{mol})$ in THF $(3 \mathrm{~mL})$ in the dark. The mixture was heated to reflux open to air in the dark. After $1.5 \mathrm{~h}$, it was cooled to room temp. and concentrated. The residue was chromatographed ( $40 \%$ to $65 \%$ ethyl acetate/hexanes, gradient) to yield $80.5 \mathrm{mg}(55 \%)$ of the title compound. $R_{f}=0.10(50 \%$ ethyl acetate/ hexanes). IR (neat): $\tilde{v}=1676(\mathrm{~m}), 1631(\mathrm{~m}), 1586(\mathrm{~m}), 1554(\mathrm{~s})$ $\mathrm{cm}^{-1} .{ }^{1} \mathrm{H}$ NMR $\left(500 \mathrm{MHz}, \mathrm{CDCl}_{3}\right): \delta=9.27(\mathrm{~s}, 1 \mathrm{H}), 9.00(\mathrm{~d}, J=$ $4.9 \mathrm{~Hz}, 1 \mathrm{H}), 7.89$ (dd, $J=4.9,0.7 \mathrm{~Hz}, 1 \mathrm{H}), 6.00(\mathrm{~s}, 1 \mathrm{H}), 5.82(\mathrm{~s}$, $1 \mathrm{H}), 4.90(\mathrm{~s}, 1 \mathrm{H}), 4.25(\mathrm{~s}, 4 \mathrm{H}) \mathrm{ppm} .{ }^{13} \mathrm{C} \mathrm{NMR}(100 \mathrm{MHz}$, $\left.\mathrm{CDCl}_{3}\right): \delta=180.43,155.53,153.78,148.15,138.44,125.11,121.16$, $118.09,118.03,105.74,58.16,57.56,39.68 \mathrm{ppm}$. MS (EI, $70 \mathrm{eV})$ : $m / z(\%)=274(53)[\mathrm{M}]^{+}, 239(100)[\mathrm{M}-\mathrm{Cl}]^{+}, 225(56)[\mathrm{M}-$ $\left.\mathrm{CH}_{2} \mathrm{Cl}\right]^{+}$. HRMS (EI, $70 \mathrm{eV}$ ): calcd. for $\mathrm{C}_{14} \mathrm{H}_{11} \mathrm{ClN}_{2} \mathrm{O}_{2}[\mathrm{M}]^{+}$ 274.0509; found 274.0504.

6-[2-(Acetoxymethyl)-2,5-dihydropyrrol-1-yl]quinoline-5,8-dione (46): Trifluoroacetic acid $(0.16 \mathrm{~mL}, 2.1 \mathrm{mmol})$ was added to 12 (50.7 mg, $0.210 \mathrm{mmol})$ in $\mathrm{CH}_{2} \mathrm{Cl}_{2}(2 \mathrm{~mL})$. After $3 \mathrm{~h}$, the mixture was concentrated and exposed to high vacuum for $1 \mathrm{~h}$. The residue was dissolved in THF $(1 \mathrm{~mL})$, and triethylamine $(32 \mu \mathrm{L}$, $0.23 \mathrm{mmol}$ ) was added. After $10 \mathrm{~min}$, this mixture was added to quinoline-5,8-dione $(33.4 \mathrm{mg}, 0.210 \mathrm{mmol})$ and cerium(III) chloride heptahydrate $(78.2 \mathrm{mg}, 0.210 \mathrm{mmol})$ in ethanol $(4 \mathrm{~mL})$ in the dark. After stirring for $18 \mathrm{~h}$ open to air in the dark, water was added, and the mixture was extracted with ethyl acetate $(5 \times)$, dried $\left(\mathrm{Na}_{2} \mathrm{SO}_{4}\right)$ and concentrated. The residue was chromatographed ( $0 \%$ to $5 \%$ methanol/ethyl acetate, gradient) to yield $33.2 \mathrm{mg}$ $(53 \%)$ of the title compound. $R_{f}=0.18(5 \%$ methanol/ethyl acetate). IR (neat): $\tilde{v}=1738$ (s), $1683(\mathrm{~m}), 1627(\mathrm{~m}), 1552$ (s) $\mathrm{cm}^{-1}$. 
${ }^{1} \mathrm{H}$ NMR $\left(400 \mathrm{MHz}, \mathrm{CDCl}_{3}\right): \delta=9.01(\mathrm{~d}, J=3.7 \mathrm{~Hz}, 1 \mathrm{H}), 8.36$ (dd, $J=8.0,1.5 \mathrm{~Hz}, 1 \mathrm{H}), 7.60(\mathrm{dd}, J=7.7,4.8 \mathrm{~Hz}, 1 \mathrm{H}), 6.05$ (s, $1 \mathrm{H}), 6.04(\mathrm{dd}, J=6.6,1.8 \mathrm{~Hz}, 1 \mathrm{H}), 5.88(\mathrm{dd}, J=6.6,2.2 \mathrm{~Hz}, 1$ $\mathrm{H}), 5.58(\mathrm{~s}, 1 \mathrm{H}), 4.42(\mathrm{~s}, 1 \mathrm{H}), 4.32-4.22(\mathrm{~m}, 1 \mathrm{H}), 4.30$ (dd, $J=$ 11.5, $2.9 \mathrm{~Hz}, 1 \mathrm{H}), 4.24(\mathrm{dd}, J=11.7,4.0 \mathrm{~Hz}, 1 \mathrm{H}), 1.98(\mathrm{~s}, 3 \mathrm{H})$ ppm. ${ }^{13} \mathrm{C}$ NMR $\left(100 \mathrm{MHz}, \mathrm{CDCl}_{3}\right): \delta=182.04,181.48,170.89$, $154.40,148.43,148.08,135.05,128.27,126.79,125.86,106.98$, $65.75,63.54,58.22,20.28 \mathrm{ppm}$. MS (EI, $70 \mathrm{eV}): \mathrm{m} / z(\%)=298(1)$ $[\mathrm{M}]^{+}, 238(100)[\mathrm{M}-\mathrm{HOAc}]^{+}$. HRMS (ESI): calcd. for $\mathrm{C}_{16} \mathrm{H}_{14} \mathrm{~N}_{2} \mathrm{O}_{4}[\mathrm{M}+\mathrm{Na}]^{+}$321.0851; found 321.0846.

6-[3-(Chloromethyl)-2,5-dihydropyrrol-1-yl]quinoline-5,8-dione (47): Trifluoroacetic acid $(0.146 \mathrm{~mL}, 1.9 \mathrm{mmol})$ was added to $\mathbf{1 0}$ (41.4 mg, $0.190 \mathrm{mmol})$ in $\mathrm{CH}_{2} \mathrm{Cl}_{2}(2 \mathrm{~mL})$. After $1.5 \mathrm{~h}$, the mixture was concentrated and exposed to high vacuum for $1 \mathrm{~h}$. The residue was dissolved in THF $(1 \mathrm{~mL})$, and triethylamine $(29.3 \mu \mathrm{L}$, $0.21 \mathrm{mmol}$ ) was added. After $10 \mathrm{~min}$, this mixture was added to quinoline-5,8-dione $(30.4 \mathrm{mg}, 0.190 \mathrm{mmol}$ ) and cerium(III) chloride heptahydrate $(70.8 \mathrm{mg}, 0.190 \mathrm{mmol})$ in ethanol $(4 \mathrm{~mL})$ in the dark. After stirring for $16 \mathrm{~h}$ open to air in the dark, water was added, and the mixture was extracted with ethyl acetate $(4 \times)$, dried $\left(\mathrm{Na}_{2} \mathrm{SO}_{4}\right)$ and concentrated. The residue was chromatographed ( $0 \%$ to $5 \%$ methanol/ethyl acetate, gradient) to yield $38.2 \mathrm{mg}$ $(73 \%)$ of the title compound. $R_{f}=0.14(5 \%$ methanol/ethyl acetate). IR (neat): $\tilde{v}=1682$ (s), 1623 (s), 1555 (s) $\mathrm{cm}^{-1} \cdot{ }^{1} \mathrm{H}$ NMR $\left(400 \mathrm{MHz}, \mathrm{CDCl}_{3}\right): \delta=8.98(\mathrm{dd}, J=4.6,1.5 \mathrm{~Hz}, 1 \mathrm{H}), 8.32(\mathrm{dd}$, $J=8.0,1.5 \mathrm{~Hz}, 1 \mathrm{H}), 7.55(\mathrm{dd}, J=8.0,4.8 \mathrm{~Hz}, 1 \mathrm{H}), 5.92(\mathrm{~s}, 2 \mathrm{H})$, 4.81 (s, $2 \mathrm{H}), 4.20$ (s, $4 \mathrm{H}) \mathrm{ppm} .{ }^{13} \mathrm{C}$ NMR $\left(100 \mathrm{MHz}, \mathrm{CDCl}_{3}\right): \delta$ $=182.18,181.05,154.85,148.77,147.56,134.62,128.10,126.32$, 106.32, 57.87, $39.58 \mathrm{ppm}$. MS (EI, $70 \mathrm{eV}): \mathrm{m} / z(\%)=274(41)$ $[\mathrm{M}]^{+}, 239(100)[\mathrm{M}-\mathrm{Cl}]^{+}, 225(58)\left[\mathrm{M}-\mathrm{CH}_{2} \mathrm{Cl}\right]^{+}$. HRMS (EI, $70 \mathrm{eV}$ ): calcd. for $\mathrm{C}_{14} \mathrm{H}_{11} \mathrm{ClN}_{2} \mathrm{O}_{2}[\mathrm{M}]^{+}$274.0509; found 274.0500 .

6-[2-(Acetoxymethyl)-4-(chloromethyl)-2,5-dihydropyrrol-1-yl]quinoline-5,8-dione (48): Trifluoroacetic acid $(0.205 \mathrm{~mL}, 2.7 \mathrm{mmol})$ was added to $13(77.0 \mathrm{mg}, 0.266 \mathrm{mmol})$ in $\mathrm{CH}_{2} \mathrm{Cl}_{2}(3 \mathrm{~mL})$. After $2.5 \mathrm{~h}$, the mixture was concentrated and exposed to high vacuum for $1 \mathrm{~h}$. The residue was dissolved in THF $(1.5 \mathrm{~mL})$, and triethylamine $(40.7 \mu \mathrm{L}, 0.292 \mathrm{mmol})$ was added. After $10 \mathrm{~min}$, this mixture was added to quinoline-5,8-dione $(42.3 \mathrm{mg}, 0.266 \mathrm{mmol})$ and cerium(III) chloride heptahydrate $(99.1 \mu \mathrm{g}, 0.266 \mathrm{mmol})$ in ethanol $(5 \mathrm{~mL})$ in the dark. After stirring for $16 \mathrm{~h}$ open to air in the dark, the mixture was diluted with ethyl acetate and washed with brine. The aqueous layer was extracted with ethyl acetate, and the combined organic layers were dried $\left(\mathrm{Na}_{2} \mathrm{SO}_{4}\right)$ and concentrated. The residue was chromatographed $(0 \%$ to $5 \%$ methanol/ethyl acetate, gradient) to yield $49.9 \mathrm{mg}(54 \%)$ of the title compound. $R_{f}=0.19$ (5\% methanol/ethyl acetate). IR (neat): $\tilde{v}=1738(\mathrm{~s}), 1683(\mathrm{~s}), 1627$ (m), $1552(\mathrm{~s}) \mathrm{cm}^{-1} \cdot{ }^{1} \mathrm{H}$ NMR $\left(400 \mathrm{MHz}, \mathrm{CDCl}_{3}\right): \delta=8.98$ (d, $J=$ $3.7 \mathrm{~Hz}, 1 \mathrm{H}), 8.33(\mathrm{dd}, J=7.9,1.5 \mathrm{~Hz}, 1 \mathrm{H}), 7.56(\mathrm{dd}, J=8.0$, $4.8 \mathrm{~Hz}, 1 \mathrm{H}), 6.03(\mathrm{~s}, 1 \mathrm{H}), 5.87(\mathrm{~s}, 1 \mathrm{H}), 5.48(\mathrm{~s}, 1 \mathrm{H}), 4.54$ (s, 1 $\mathrm{H}), 4.52-4.17(\mathrm{~m}, 1 \mathrm{H}), 4.32(\mathrm{dd}, J=11.5,2.9 \mathrm{~Hz}, 1 \mathrm{H}), 4.23(\mathrm{dd}$, $J=11.7,4.4 \mathrm{~Hz}, 1 \mathrm{H}), 4.20(\mathrm{~s}, 2 \mathrm{H}), 1.98(\mathrm{~s}, 3 \mathrm{H}) \mathrm{ppm} .{ }^{13} \mathrm{C} \mathrm{NMR}$ $\left(100 \mathrm{MHz}, \mathrm{CDCl}_{3}\right): \delta=183.12,181.96,171.12,155.47,148.92$, 148.18, $135.30129 .00,127.08,126.07,108.74,66.80,64.00,58.79$, 39.90, $21.07 \mathrm{ppm}$. MS (EI, $70 \mathrm{eV}): m / z(\%)=237(59)[\mathrm{M}-\mathrm{H}-$ $\left.\mathrm{CH}_{2} \mathrm{OAc}-\mathrm{Cl}\right]^{+}, 43(100)\left[\mathrm{COCH}_{3}\right]^{+}$. HRMS (ESI): calcd. for $\mathrm{C}_{17} \mathrm{H}_{15} \mathrm{ClN}_{2} \mathrm{O}_{4}[\mathrm{M}+\mathrm{Na}]^{+}$369.0618; found 369.0607.

6-[cis-2,5-Bis(acetoxymethyl)-2,5-dihydropyrrol-1-yl]quinoline-5,8-dione (49): Trifluoroacetic acid $(0.223 \mathrm{~mL}, 2.9 \mathrm{mmol})$ was added to 14 (92.1 mg, $0.290 \mathrm{mmol}$ ) in $\mathrm{CH}_{2} \mathrm{Cl}_{2}(3 \mathrm{~mL})$. After $2.5 \mathrm{~h}$, the reaction was not complete, so an additional portion of trifluoroacetic acid $(50 \mu \mathrm{L}, 0.65 \mathrm{mmol})$ was added. After $3 \mathrm{~h}$, the mixture was concentrated and exposed to high vacuum for $1 \mathrm{~h}$. The residue was dissolved in THF $(1.5 \mathrm{~mL})$, and triethylamine $(44.6 \mu \mathrm{L}, 0.32 \mathrm{mmol})$ was added. After $10 \mathrm{~min}$, this mixture was added to quinoline-5,8dione (46.8 mg, $0.290 \mathrm{mmol}$ ) and cerium(III) chloride heptahydrate $(0.108 \mathrm{~g}, 0.290 \mathrm{mmol})$ in ethanol $(6 \mathrm{~mL})$ in the dark. After stirring for $2 \mathrm{~d}$ open to air in the dark, water and brine were added, and the mixture was extracted with ethyl acetate $(4 \times)$, dried $\left(\mathrm{Na}_{2} \mathrm{SO}_{4}\right)$ and concentrated. The residue was chromatographed $(0 \%$ to $5 \%$ methanol/ethyl acetate, gradient) to yield $40.6 \mathrm{mg} \mathrm{(38 \% )}$ of the title compound. $R_{f}=0.20$ (5\% methanol/ethyl acetate). IR (neat): $\tilde{v}=$ 1742 (s), 1684 (s), 1547 (s) $\mathrm{cm}^{-1} \cdot{ }^{1} \mathrm{H}$ NMR (400 MHz, $\left.\mathrm{CDCl}_{3}\right): \delta=$ $8.98(\mathrm{dd}, J=4.8,1.5 \mathrm{~Hz}, 1 \mathrm{H}), 8.34(\mathrm{dd}, J=7.9,1.5 \mathrm{~Hz}, 1 \mathrm{H}), 7.58$ $(\mathrm{dd}, J=8.0,4.8 \mathrm{~Hz}, 1 \mathrm{H}), 6.25(\mathrm{~s}, 1 \mathrm{H}), 5.99(\mathrm{~s}, 1 \mathrm{H}), 5.79(\mathrm{~s}, 1 \mathrm{H})$, $5.16(\mathrm{t}, J=3.7 \mathrm{~Hz}, 1 \mathrm{H}), 4.43(\mathrm{dd}, J=11.3,3.7 \mathrm{~Hz}, 1 \mathrm{H}), 4.36(\mathrm{t}$, $J=4.8 \mathrm{~Hz}, 1 \mathrm{H}), 4.23(\mathrm{dd}, J=11.3,5.9 \mathrm{~Hz}, 1 \mathrm{H}), 4.00(\mathrm{dd}, J=$ 9.5, 5.3 Hz, $1 \mathrm{H}), 2.09(\mathrm{~s}, 3 \mathrm{H}), 2.05(\mathrm{~s}, 3 \mathrm{H}) \mathrm{ppm} .{ }^{13} \mathrm{C} \mathrm{NMR}$ $\left(100 \mathrm{MHz}, \mathrm{CDCl}_{3}\right): \delta=181.85,171.18,170.66,154.84,147.67$, 135.13, 128.28, 127.76, 126.88, 107.72, 93.99, 66.87, 64.97, 64.05, $62.53,20.71,20.37 \mathrm{ppm}$. MS (EI, $70 \mathrm{eV}): m / z(\%)=252(100)[\mathrm{M}-$ $2 \mathrm{OAc}^{+}$. HRMS (ESI): calcd. for $\mathrm{C}_{19} \mathrm{H}_{18} \mathrm{~N}_{2} \mathrm{O}_{6}[\mathrm{M}+\mathrm{Na}]^{+}$ 393.1063; found 393.1068.

2-[3-(Hydroxymethyl)pyrrol-1-yl]-1,4-naphthoquinone (52): An NMR sample of the pyrroline 34 in $\mathrm{CDCl}_{3}$ was allowed to stand under ambient laboratory light until the starting material was consumed (approximately $3 \mathrm{~d}): R_{f}=0.17\left(30 \%\right.$ acetone/hexanes). ${ }^{1} \mathrm{H}$ NMR ( $\left.\mathrm{CDCl}_{3}, 500 \mathrm{MHz}\right): \delta=8.20-8.11(\mathrm{~m}, 2 \mathrm{H}), 7.83-7.76(\mathrm{~m}, 2$ H), 7.31-7.29 (m, 1 H), 7.27-7.25 (m, $1 \mathrm{H}), 6.82$ (s, $1 \mathrm{H}), 6.46-6.45$ $(\mathrm{m}, 1 \mathrm{H}), 4.63(\mathrm{~s}, 2 \mathrm{H}), 1.25(\mathrm{~s}, 1 \mathrm{H}) \mathrm{ppm} .{ }^{13} \mathrm{C} \mathrm{NMR}\left(\mathrm{CDCl}_{3}\right.$, $125 \mathrm{MHz}): \delta=184.4,181.1,142.7,134.5,133.9,131.5,131.7,128.9$, $127.2,126.1,122.5,122.4,119.6,112.6,58.6 \mathrm{ppm}$. UV $\left(\mathrm{CHCl}_{3}\right)$ : $\lambda_{\text {max }}=257,307,643,400 \mathrm{~nm}(\log \varepsilon=4.17,3.90,3.45,3.69)$.

2-\{3-[(Ethylsulfanyl)methyl]pyrrol-1-yl\}-1,4-naphthoquinone (53): Ethanethiol $(24 \mu \mathrm{L}, 0.32 \mathrm{mmol})$ was added to $36(8.80 \mathrm{mg}$, $32.0 \mu \mathrm{mol})$ and 2,6 -lutidine $(3.7 \mu \mathrm{L}, 32 \mu \mathrm{mol})$ in chloroform $(2 \mathrm{~mL})$. After being exposed to ambient light and open to air for $2 \mathrm{~d}$, there was no 36 remaining so the mixture was adsorbed on silica gel, while the solvent was removed in a rotavapor, and chromatographed (10\% to $20 \%$ ethyl acetate/hexanes, gradient) to yield $6.0 \mathrm{mg}(63 \%)$ of the title compound. $R_{f}=0.34(20 \%$ ethyl acetate/ hexanes). IR (neat): $\tilde{v}=1679$ (s), 1646 (s) $\mathrm{cm}^{-1} \cdot{ }^{1} \mathrm{H} \mathrm{NMR}$ $\left(400 \mathrm{MHz}, \mathrm{CDCl}_{3}\right): \delta=8.14-8.12(\mathrm{~m}, 1 \mathrm{H}), 8.08-8.06(\mathrm{~m}, 1 \mathrm{H})$, 7.76-7.73 (m, $2 \mathrm{H}), 7.24$ (s, $1 \mathrm{H}), 7.19$ (s, $1 \mathrm{H}), 6.76$ (s, $1 \mathrm{H}), 6.36$ (s, $1 \mathrm{H}), 3.61$ (s, $2 \mathrm{H}), 2.48$ (q, $J=7.3 \mathrm{~Hz}, 2 \mathrm{H}), 1.23(\mathrm{t}, J=7.3 \mathrm{~Hz}$, $3 \mathrm{H}) \mathrm{ppm} .{ }^{13} \mathrm{C} \mathrm{NMR}\left(100 \mathrm{MHz}, \mathrm{CDCl}_{3}\right): \delta=184.41,181.05$, $142.58,134.40,133.80,131.98,131.52,127.16,126.01,125.38$, $122.30,121.91,119.65,113.77,27.70,25.38,14.40$ ppm. MS (EI, $70 \mathrm{eV}): \mathrm{m} / z(\%)=297(17)[\mathrm{M}]^{+}, 236(100)[\mathrm{M}-\mathrm{SEt}]^{+}$. HRMS (EI, $70 \mathrm{eV}$ ): calcd. for $\mathrm{C}_{17} \mathrm{H}_{15} \mathrm{NO}_{2} \mathrm{~S}[\mathrm{M}-\mathrm{H}]^{+}$296.0745; found 296.0747 .

2-\{2-[(Ethylsulfanyl)methyl]pyrrol-1-yl\}-1,4-naphthoquinone (55): Ethanethiol $(26 \mu \mathrm{L}, 0.35 \mathrm{mmol})$ was added to $38(10.3 \mathrm{mg}$, $35.0 \mu \mathrm{mol})$ in chloroform $(3 \mathrm{~mL})$. After being exposed to ambient light and open to air for $24 \mathrm{~h}$, there was no $\mathbf{3 8}$ remaining so the mixture was adsorbed on silica gel, while the solvent was removed in a rotavapor, and chromatographed (10\% to $20 \%$ ethyl acetate/ hexanes, gradient) to yield a mixture of quinone and hydroquinone products. The mixture was dissolved in $\mathrm{CH}_{2} \mathrm{Cl}_{2}$, and silica gel was added. After stirring for $2 \mathrm{~h}$, the only product present was the quinone product. Filtration and concentration yielded $9.0 \mathrm{mg}(86 \%)$ of the title compound. $R_{f}=0.22(10 \%$ ethyl acetate/hexanes). IR (neat): $\tilde{v}=1678$ (s), 1659 (s), $1280(\mathrm{~s}) \mathrm{cm}^{-1} \cdot{ }^{1} \mathrm{H}$ NMR $(400 \mathrm{MHz}$, $\left.\mathrm{CDCl}_{3}\right): \delta=8.16(\mathrm{dd}, J=5.8,3.5 \mathrm{~Hz}, 1 \mathrm{H}), 8.12(\mathrm{dd}, J=5.6$, $3.5 \mathrm{~Hz}, 1 \mathrm{H}), 7.78(\mathrm{dd}, J=5.1,4.0 \mathrm{~Hz}, 2 \mathrm{H}), 6.85(\mathrm{~s}, 1 \mathrm{H}), 6.78$ (dd, $J=2.9,1.8 \mathrm{~Hz}, 1 \mathrm{H}), 6.24-6.21(\mathrm{~m}, 2 \mathrm{H}), 3.78(\mathrm{~s}, 2 \mathrm{H}), 2.20$ 
(q, $J=7.3 \mathrm{~Hz}, 2 \mathrm{H}), 1.06(\mathrm{t}, J=7.3 \mathrm{~Hz}, 3 \mathrm{H}) \mathrm{ppm} .{ }^{13} \mathrm{C} \mathrm{NMR}$ $\left(100 \mathrm{MHz}, \mathrm{CDCl}_{3}\right): \delta=184.85,180.23,145.94,134.22,134.04$, 131.98, 130.00, 127.91, 127.21, 126.33, 123.88, 121.98, 113.34, 109.96, 28.04, 25.01, $13.95 \mathrm{ppm}$. MS (EI, $70 \mathrm{eV}): \mathrm{m} / \mathrm{z}(\%)=297$ (15) $[\mathrm{M}]^{+}, 236(100)[\mathrm{M}-\mathrm{SEt}]^{+}$. HRMS (EI, $\left.70 \mathrm{eV}\right)$ : calcd. for $\mathrm{C}_{17} \mathrm{H}_{15} \mathrm{NO}_{2} \mathrm{~S}[\mathrm{M}]^{+}$297.0824; found 297.0833.

6-\{3-I(Ethylsulfanyl)methyl|pyrrol-1-yl\}quinoline-5,8-diol (56): Ethanethiol $(52 \mu \mathrm{L}, 0.70 \mathrm{mmol})$ was added to $47(19.3 \mathrm{mg}, 70.0 \mu \mathrm{mol})$ and 2,6-lutidine $(8.2 \mu \mathrm{L}, 70 \mu \mathrm{mol})$ in chloroform $(3.5 \mathrm{~mL})$. After being exposed to ambient light and open to air for $18 \mathrm{~h}$, there was no 47 remaining so the mixture was adsorbed on silica gel, while the solvent was removed in a rotavapor, and chromatographed ( $20 \%$ to $30 \%$ ethyl acetate/hexanes, gradient) to yield $8.8 \mathrm{mg} \mathrm{(42 \% )}$ of the title compound. $R_{f}=0.19$ (20\% ethyl acetate/hexanes). IR (neat): $\tilde{v}=3390$ (br.) $\mathrm{cm}^{-1} .{ }^{1} \mathrm{H}$ NMR $\left(300 \mathrm{MHz}, \mathrm{CDCl}_{3}\right): \delta=8.81$ $(\mathrm{dd}, J=4.4,1.3 \mathrm{~Hz}, 1 \mathrm{H}), 8.58(\mathrm{dd}, J=7.8,1.3 \mathrm{~Hz}, 1 \mathrm{H}), 7.50(\mathrm{dd}$, $J=8.5,4.4 \mathrm{~Hz}, 1 \mathrm{H}), 7.05(\mathrm{~s}, 1 \mathrm{H}), 6.88-6.87(\mathrm{~m}, 2 \mathrm{H}), 6.41(\mathrm{t}, J$ $=1.9 \mathrm{~Hz}, 1 \mathrm{H}), 3.72(\mathrm{~s}, 2 \mathrm{H}), 2.57(\mathrm{q}, J=7.4 \mathrm{~Hz}, 2 \mathrm{H}), 1.29(\mathrm{t}, J$ $=7.4 \mathrm{~Hz}, 3 \mathrm{H}) \mathrm{ppm} \cdot{ }^{13} \mathrm{C} \mathrm{NMR}\left(100 \mathrm{MHz}, \mathrm{CDCl}_{3}\right): \delta=148.59$, $146.17,137.44,131.88,123.44,122.71,122.18,121.73,120.10$, 111.64, 107.89, 29.62, 27.80, 25.63, $14.38 \mathrm{ppm}$. MS (EI, $70 \mathrm{eV}): \mathrm{m} / \mathrm{z}$ $(\%)=239(100)[\mathrm{M}-\mathrm{SEt}]^{+}, 238(99)[\mathrm{M}-\mathrm{HSEt}]^{+}$. HRMS (ESI): calcd. for $\mathrm{C}_{16} \mathrm{H}_{16} \mathrm{~N}_{2} \mathrm{O}_{2} \mathrm{~S}[\mathrm{M}+\mathrm{H}]^{+}$301.1011; found 301.1010.

6-\{2-I(Ethylsulfanyl)methyl|pyrrol-1-yl\}quinoline-5,8-diol (57): Ethanethiol $(21 \mu \mathrm{L}, 0.28 \mathrm{mmol})$ was added to $46(8.4 \mathrm{mg}, 28 \mu \mathrm{mol})$ in chloroform $(3 \mathrm{~mL})$. After being exposed to ambient light and open to air for $16 \mathrm{~h}$, there was no 46 remaining so the mixture was adsorbed on silica gel, while the solvent was removed in a rotavapor, and chromatographed (20\% ethyl acetate/hexanes) to yield $5.6 \mathrm{mg}$ $(67 \%)$ of the title compound. $R_{f}=0.25(30 \%$ ethyl acetate/hexanes). IR (neat): $\tilde{v}=3398$ (br.) cm $\left.{ }^{-1} .{ }^{1} \mathrm{H} \mathrm{NMR} \mathrm{(300} \mathrm{MHz,} \mathrm{CDCl}_{3}\right)$ : $\delta=8.85(\mathrm{dd}, J=4.2,1.6 \mathrm{~Hz}, 1 \mathrm{H}), 8.64(\mathrm{dd}, J=8.4,1.6 \mathrm{~Hz}, 1 \mathrm{H})$, 7.88 (s, $1 \mathrm{H}), 7.52(\mathrm{dd}, J=8.5,4.1 \mathrm{~Hz}, 1 \mathrm{H}), 6.97(\mathrm{~s}, 1 \mathrm{H}), 6.75$ $(\mathrm{dd}, J=2.7,1.9 \mathrm{~Hz}, 1 \mathrm{H}), 6.30(\mathrm{dd}, J=3.6,2.7 \mathrm{~Hz}, 1 \mathrm{H}), 6.26(\mathrm{dd}$, $J=3.6,1.6 \mathrm{~Hz}, 1 \mathrm{H}), 5.97(\mathrm{~s}, 1 \mathrm{H}), 3.64(\mathrm{~s}, 1 \mathrm{H}), 3.52$ (s, $1 \mathrm{H}), 2.45$ (q, $J=7.4 \mathrm{~Hz}, 2 \mathrm{H}), 1.16(\mathrm{t}, J=7.4 \mathrm{~Hz}, 3 \mathrm{H}) \mathrm{ppm} .{ }^{13} \mathrm{C}$ NMR $\left(100 \mathrm{MHz}, \mathrm{CDCl}_{3}\right): \delta=149.03,146.24,140.19,138.49,132.36$, $130.37,123.55,122.02,121.70,120.80,110.00,109.83,109.52$, $26.85,25.50,14.12 \mathrm{ppm}$. MS (EI, $70 \mathrm{eV}): \mathrm{m} / z(\%)=238(100)[\mathrm{M}-$ HSEt ${ }^{+}$. HRMS (ESI): calcd. for $\mathrm{C}_{16} \mathrm{H}_{16} \mathrm{~N}_{2} \mathrm{O}_{2} \mathrm{~S}[\mathrm{M}+\mathrm{H}]^{+} 301.1011$; found 301.1008 .

2-\{2-[(Ethylsulfanyl)methyl|pyrrol-1-yl\}-8-hydroxy-1,4-naphthoquinone (58): Ethanethiol $(24 \mu \mathrm{L}, 0.32 \mathrm{mmol})$ was added to $42(10.2 \mathrm{mg}$, $32.0 \mu \mathrm{mol})$ in chloroform $(1.6 \mathrm{~mL})$. After being exposed to ambient light and open to air for $2 \mathrm{~d}$, there was no $\mathbf{4 2}$ remaining so the mixture was adsorbed on silica gel, while the solvent was removed in a rotavapor, and chromatographed (10\% to $20 \%$ ethyl acetate/ hexanes, gradient) to yield $4.4 \mathrm{mg}(44 \%)$ of the title compound. $R_{f}$ $=0.47$ (30\% ethyl acetate/hexanes). IR (neat): $\tilde{v}=1642(\mathrm{~s}), 1606$ (s), 1277 (s) $\mathrm{cm}^{-1} \cdot{ }^{1} \mathrm{H}$ NMR (400 MHz, $\left.\mathrm{CDCl}_{3}\right): \delta=11.73(\mathrm{~s}, 1 \mathrm{H})$, $7.65(\mathrm{~d}, J=4.4 \mathrm{~Hz}, 2 \mathrm{H}), 7.29(\mathrm{t}, J=4.8 \mathrm{~Hz}, 1 \mathrm{H}), 6.85(\mathrm{~s}, 1 \mathrm{H})$, $6.75(\mathrm{t}, J=2.9 \mathrm{~Hz}, 1 \mathrm{H}), 6.22-6.20(\mathrm{~m}, 2 \mathrm{H}), 3.76(\mathrm{~s}, 2 \mathrm{H}), 2.20$ (q, $J$ $=7.3 \mathrm{~Hz}, 2 \mathrm{H}), 1.05(\mathrm{t}, J=7.3 \mathrm{~Hz}, 3 \mathrm{H}) \mathrm{ppm} .{ }^{13} \mathrm{C} \mathrm{NMR}(100 \mathrm{MHz}$, $\left.\mathrm{CDCl}_{3}\right): \delta=184.58,162.76,137.71,132.71,131.70,130.58,125.35$, $124.73,119.89,115.34,113.79,110.52,94.62,28.42,25.57$, $14.60 \mathrm{ppm}$. MS (EI, $70 \mathrm{eV}): \mathrm{m} / z(\%)=313(19)[\mathrm{M}]^{+}, 252(100)$ $[\mathrm{M}-\mathrm{SEt}]^{+}$. HRMS (EI, $70 \mathrm{eV}$ ): calcd. for $\mathrm{C}_{17} \mathrm{H}_{15} \mathrm{NO}_{3} \mathrm{~S}[\mathrm{M}]^{+}$ 313.0773; found 313.0775 .

2-\{2,4-Bis[(ethylsulfanyl)methyl]pyrrol-1-yl\}naphthalene-1,4-diol (59): Ethanethiol $(120 \mu \mathrm{L}, 1.6 \mathrm{mmol})$ was added to $39(53.8 \mathrm{mg}$, $160 \mu \mathrm{mol})$ and 2,6 -lutidine $(18 \mu \mathrm{L}, 0.16 \mathrm{mmol})$ in acetonitrile $(8 \mathrm{~mL})$. After being exposed to ambient light and open to air for
$2 \mathrm{~d}$, there was no 39 remaining so the mixture was adsorbed on silica gel, while the solvent was removed in a rotavapor, and chromatographed (10\% to $20 \%$ ethyl acetate/hexanes, gradient) to yield $45.2 \mathrm{mg}(76 \%)$ of the title compound. Although characterized as the hydroquinone, this compound was found to be relatively unstable, converting over time to the quinone product. $R_{f}=0.38(30 \%$ ethyl acetate/hexanes). IR (neat): $\tilde{v}=3388$ (br.) $\mathrm{cm}^{-1}$. ${ }^{1} \mathrm{H}$ NMR $\left(300 \mathrm{MHz}, \mathrm{CDCl}_{3}\right): \delta=8.28(\mathrm{dd}, J=6.2,3.3 \mathrm{~Hz}, 1 \mathrm{H}), 8.16(\mathrm{dd}$, $J=5.9,3.3 \mathrm{~Hz}, 1 \mathrm{H}), 7.58(\mathrm{dd}, J=6.3,3.3 \mathrm{~Hz}, 2 \mathrm{H}), 6.64(\mathrm{~s}, 2 \mathrm{H})$, $6.23(\mathrm{~s}, 1 \mathrm{H}), 5.77(\mathrm{~s}, 2 \mathrm{H}), 3.65(\mathrm{~s}, 4 \mathrm{H}), 2.55(\mathrm{q}, J=7.4 \mathrm{~Hz}, 2 \mathrm{H})$, $2.45(\mathrm{q}, J=7.4 \mathrm{~Hz}, 2 \mathrm{H}), 1.27(\mathrm{t}, J=7.4 \mathrm{~Hz}, 3 \mathrm{H}), 1.15(\mathrm{t}, J=$ $7.4 \mathrm{~Hz}, 3 \mathrm{H}) \mathrm{ppm} .{ }^{13} \mathrm{C} \mathrm{NMR}\left(100 \mathrm{MHz}, \mathrm{CDCl}_{3}\right): \delta=145.10$, $141.97,130.88,126.64,126.60,125.93,125.35,122.98,121.88$, $121.68,121.40,119.69,110.25,108.60,27.87,26.63,25.61,25.49$, $14.43,14.10 \mathrm{ppm}$. MS (EI, $70 \mathrm{eV}): m / z(\%)=311(63)[\mathrm{M}-$ HSEt $]^{+}, 250$ (100) $[\mathrm{M}-\mathrm{HSEt}-\mathrm{SEt}]^{+}$. HRMS (ESI): calcd. for $\mathrm{C}_{20} \mathrm{H}_{23} \mathrm{NO}_{2} \mathrm{~S}_{2}[\mathrm{M}+\mathrm{Na}]^{+}$396.1068; found 396.1068.

2-\{2,5-Bis[(ethylsulfanyl)methyl]pyrrol-1-yl\}naphthalene-1,4-diol (60): Ethanethiol $(47 \mu \mathrm{L}, 0.63 \mathrm{mmol})$ was added to $40(23.3 \mathrm{mg}$, $63.0 \mu \mathrm{mol})$ in chloroform $(3 \mathrm{~mL})$. After being exposed to ambient light and open to air for $2 \mathrm{~d}$, there was no starting material remaining so the mixture was adsorbed onto silica gel and chromatographed $(10 \%$ to $20 \%$ ethyl acetate/hexanes, gradient) to yield $19.0 \mathrm{mg}(81 \%)$ of the title compound. $R_{f}=0.29(20 \%$ ethyl acetate/ hexanes). IR (neat): $\tilde{v}=3391$ (br.), 1598 (s) $\mathrm{cm}^{-1} \cdot{ }^{1} \mathrm{H}$ NMR (400 MHz, $\left.\mathrm{CDCl}_{3}\right): \delta=8.32-8.29(\mathrm{~m}, 1 \mathrm{H}), 8.18-8.15(\mathrm{~m}, 1 \mathrm{H})$, $7.57(\mathrm{td}, J=4.9,1.5 \mathrm{~Hz}, 2 \mathrm{H}), 6.60(\mathrm{~s}, 1 \mathrm{H}), 6.15(\mathrm{~s}, 2 \mathrm{H}), 5.80(\mathrm{~s}$ $1 \mathrm{H}), 5.32(\mathrm{~s}, 1 \mathrm{H}), 3.44(\mathrm{~d}, J=2.9 \mathrm{~Hz}, 4 \mathrm{H}), 2.40(\mathrm{q}, J=7.3 \mathrm{~Hz}$, $4 \mathrm{H}), 1.11$ (t, $J=7.3 \mathrm{~Hz}, 6 \mathrm{H}) \mathrm{ppm} .{ }^{13} \mathrm{C} \mathrm{NMR}\left(100 \mathrm{MHz}, \mathrm{CDCl}_{3}\right)$ : $\delta=145.28,142.92,130.98,126.77,126.60,125.70,123.24,121.69$, $118.27,109.43,108.70,27.10,25.50,14.16 \mathrm{ppm}$. MS (EI, $70 \mathrm{eV})$ $\mathrm{m} / \mathrm{z}(\%)=311(66)[\mathrm{M}-\mathrm{HSEt}]^{+}, 250(100)[\mathrm{M}-\mathrm{HSEt}-\mathrm{SEt}]^{+}$ HRMS (ESI): calcd. for $\mathrm{C}_{20} \mathrm{H}_{23} \mathrm{NO}_{2} \mathrm{~S}_{2}[\mathrm{M}+\mathrm{Na}]^{+} 396.1068$; found 396.1082

6-\{2,4-Bis[(ethylsulfanyl)methyl]pyrrol-1-yl\}quinoline-5,8-diol (61): Ethanethiol $(47 \mu \mathrm{L}, 0.64 \mathrm{mmol})$ was added to $48(22.3 \mathrm{mg}$, $64.0 \mu \mathrm{mol})$ in chloroform $(3 \mathrm{~mL})$. After being exposed to ambient light and open to air for $18 \mathrm{~h}$, there was no $\mathbf{4 8}$ remaining so the mixture was adsorbed on silica gel, while the solvent was removed in a rotavapor, and chromatographed $(20 \%$ to $30 \%$ ethyl acetate/ hexanes, gradient) to yield $10.6 \mathrm{mg}(44 \%)$ of the title compound. $R_{f}=0.14$ (20\% ethyl acetate/hexanes). IR (neat): $\tilde{v}=3390$ (br.) $\mathrm{cm}^{-1} .{ }^{1} \mathrm{H}$ NMR $\left(300 \mathrm{MHz}, \mathrm{CDCl}_{3}\right): \delta=8.85(\mathrm{dd}, J=4.1,1.4 \mathrm{~Hz}$, $1 \mathrm{H}), 8.63(\mathrm{dd}, J=8.5,1.4 \mathrm{~Hz}, 1 \mathrm{H}), 7.87(\mathrm{~s}, 1 \mathrm{H}), 7.52(\mathrm{dd}, J=$ $8.5,4.1 \mathrm{~Hz}, 1 \mathrm{H}), 6.95(\mathrm{~s}, 1 \mathrm{H}), 6.66(\mathrm{~d}, J=1.6 \mathrm{~Hz}, 1 \mathrm{H}), 6.24(\mathrm{~d}$, $J=1.6 \mathrm{~Hz}, 1 \mathrm{H}), 6.02(\mathrm{~s}, 1 \mathrm{H}), 3.66(\mathrm{~s}, 4 \mathrm{H}), 2.55(\mathrm{q}, J=7.4 \mathrm{~Hz}$, $2 \mathrm{H}), 2.46(\mathrm{q}, J=7.4 \mathrm{~Hz}, 2 \mathrm{H}), 1.27(\mathrm{t}, J=7.4 \mathrm{~Hz}, 3 \mathrm{H}), 1.16(\mathrm{t}$, $J=7.4 \mathrm{~Hz}, 3 \mathrm{H}) \mathrm{ppm} .{ }^{13} \mathrm{C} \mathrm{NMR}\left(100 \mathrm{MHz}, \mathrm{CDCl}_{3}\right): \delta=149.03$, $146.26,140.16,138.48,132.35,130.94,121.99,121.86,121.72$, $120.82,110.62,109.79,27.97,26.88,25.64,25.55,14.46,14.14$ ppm. MS (EI, $70 \mathrm{eV}): m / z(\%)=312(53)[\mathrm{M}-\mathrm{HSEt}]^{+}, 251(100)[\mathrm{M}-$ $\mathrm{HSEt}-\mathrm{SEt}]^{+}$. HRMS (ESI): calcd. for $\mathrm{C}_{19} \mathrm{H}_{22} \mathrm{~N}_{2} \mathrm{O}_{2} \mathrm{~S}_{2}[\mathrm{M}+\mathrm{H}]^{+}$ 375.1201; found 375.1199 .

6-\{2,5-Bis[(ethylsulfanyl)methyl]pyrrol-1-yl\}quinoline-5,8-diol (62): Ethanethiol $(17 \mu \mathrm{L}, 0.23 \mathrm{mmol})$ was added to $49(8.5 \mathrm{mg}, 23 \mu \mathrm{mol})$ in chloroform $(1 \mathrm{~mL})$. After being exposed to ambient light and open to air for $16 \mathrm{~h}$, there was no 49 remaining so the mixture was adsorbed on silica gel, while the solvent was removed in a rotavapor, and chromatographed (50\% ethyl acetate/hexanes) to yield $2.6 \mathrm{mg}(30 \%)$ of the title compound. $R_{f}=0.17$ (30\% ethyl acetate/ hexanes). IR (neat): $\tilde{v}=3392$ (br.) $\mathrm{cm}^{-1} \cdot{ }^{1} \mathrm{H}$ NMR $(400 \mathrm{MHz}$, $\left.\mathrm{CDCl}_{3}\right): \delta=8.86(\mathrm{dd}, J=4.4,1.5 \mathrm{~Hz}, 1 \mathrm{H}), 8.69(\mathrm{dd}, J=8.4$, 
$1.5 \mathrm{~Hz}, 1 \mathrm{H}), 7.54$ (dd, $J=8.4,4.4 \mathrm{~Hz}, 1 \mathrm{H}), 6.95(\mathrm{~s}, 1 \mathrm{H}), 6.16(\mathrm{~s}$, $2 \mathrm{H}), 6.06(\mathrm{~s}, 1 \mathrm{H}), 3.52(\mathrm{~d}, J=14.6 \mathrm{~Hz}, 2 \mathrm{H}), 3.40(\mathrm{~d}, J=14.3 \mathrm{~Hz}$, $2 \mathrm{H}), 2.41$ (q, $J=7.3 \mathrm{~Hz}, 4 \mathrm{H}), 1.11(\mathrm{t}, J=7.3 \mathrm{~Hz}, 6 \mathrm{H}) \mathrm{ppm} .{ }^{13} \mathrm{C}$ NMR $\left(100 \mathrm{MHz}, \mathrm{CDCl}_{3}\right): \delta=148.73,146.15,142.50,141.10$, 133.29, 131.03, 121.94, 121.63, 120.81, 111.26, 108.95, 27.14, 25.39, 14.16 ppm. MS (EI, $70 \mathrm{eV}): m / z(\%)=312(32)[\mathrm{M}-\mathrm{HSEt}]^{+}, 251$ (100) $[\mathrm{M}-\mathrm{HSEt}-\mathrm{SEt}]^{+}$. HRMS (ESI): calcd. for $\mathrm{C}_{19} \mathrm{H}_{22} \mathrm{~N}_{2} \mathrm{O}_{2} \mathrm{~S}_{2}$ $[\mathrm{M}+\mathrm{H}]^{+}$375.1201; found 375.1184.

Supporting Information (see footnote on the first page of this article): Spectra for all new compounds.

\section{Acknowledgments}

We thank Trisha Duffey for helpful experimental work on the 3pyrroline syntheses. A. A. gratefully acknowledges the Eastman Kodak Corporation and the American Chemical Society Division of Organic Chemistry sponsored by the Schering-Plough Research Institute for graduate fellowships.

[1] For recent reviews, see: a) S. R. Rajski, R. M. Williams, Chem. Rev. 1998, 98, 2732; b) D. M. Noll, T. M. Mason, P. S. Miller, Chem. Rev. 2006, 106, 277.

[2] For isolation of hepatotoxic pyrrolizidine alkaloids, see: L. W. Smith, C. C. J. Culvenor, J. Nat. Prod. 1981, 44, 129.

[3] H. Niwa, T. Ogawa, K. Yamada, Tetrahedron Lett. 1991, 32, 927.

[4] J. S. Woo, S. T. Sigurdsson, P. B. Hopkins, J. Am. Chem. Soc. 1993, 115, 3407.

[5] a) W. H. Pearson, S. C. Bergmeier, J. A. Chytra, Synthesis 1990, 156-159; b) W. K. Anderson, D. Bhattacharjee, D. M. Houston, J. Med. Chem. 1989, 32, 119; c) W. K. Anderson, H. L. McPherson, J. S. New, A. C. Rick, J. Med. Chem. 1984, 27,
1321; d) W. K. Anderson, C. P. Chang, H. L. McPherson, J. Med. Chem. 1983, 26, 1333; e) W. K. Anderson, H. L. McPherson, J. Med. Chem. 1982, 25, 84; f) W. K. Anderson, M. J. Halat, A. C. Rick, J. Med. Chem. 1980, 23, 87; g) W. K. Anderson, M. J. Halat, J. Med. Chem. 1979, 22, 977; h) W. K. Anderson, P. F. Corey, J. Med. Chem. 1977, 20, 812; i) W. K. Anderson, P. F. Corey, J. Med. Chem. 1977, 20, 1691; j) See ref. ${ }^{[4]}$; k) S. T. Sigurdsson, P. B. Hopkins, Tetrahedron 1994, 50, 12065; 1) M. F. Weidner, S. T. Sigurdsson, P. B. Hopkins, Biochemistry 1990, 29, 9225; m) See ref. ${ }^{[3]}$; n) R. H. Barbour, D. J. Robins, J. Chem. Soc. Perkin Trans. 1 1985, 2475; o) A. R. Mattocks, J. Chem. Soc. Perkin Trans. 1 1978, 896; p) D. Laduree, J. C. Lancelot, M. Robba, E. Chenu, G. Mathe, J. Med. Chem. 1989, 32, 456; q) I. Lalezari, E. L. Schwartz, J. Med. Chem. $1988,31,1427$

[6] J. J. Tepe, R. M. Williams, J. Am. Chem. Soc. 1999, 121, 2951.

[7] T. C. Judd, R. M. Williams, Org. Lett. 2002, 4, 3711.

[8] a) K. Maruyama, T. Kozuka, T. Otsuki, Bull. Chem. Soc. Jpn. 1977, 50, 2170; b) K. Maruyama, T. Kozuka, T. Otsuki, Y. Naruta, Chem. Lett. 1977, 1125; c) K. J. Falci, R. W. Franck, G. P. Smith, J. Org. Chem. 1977, 42, 3317.

[9] B. Armitage, Chem. Rev. 1998, 98, 1171.

[10] Handbook of Metathesis (Eds.: R. H. Grubbs), John Wiley \& Sons, New York, USA, 2003.

[11] C. M. Huwe, S. Blechert, Synthesis 1997, 61.

[12] A. Afzali-Ardakani, H. Rapoport, J. Org. Chem. 1980, 45, 4817.

[13] T. J. Donohoe, C. E. Headley, R. P. C. Cousins, A. Cowley, Org. Lett. 2003, 5, 999.

[14] For Cu-catalyzed Michael addition of amines to quinones, see: V. V. Russkikh, Russ. J. Org. Chem. 1995, 31, 343.

[15] A. D. Abell, B. K. Nabbs, A. R. Battersby, J. Am. Chem. Soc. 1998, 120, 1741.

Received: May 16, 2008 Published Online: July 9, 2008 